\title{
Control of flow with trapped vortices: theory and experiments
}

\author{
O.R. Tutty ${ }^{\star}$ \\ M. Buffoni ${ }^{\dagger}$ \\ R. Kerminbekov ${ }^{\star}$ \\ R. Donelli ${ }^{+}$ \\ F. De Gregorio ${ }^{+}$ \\ E. Rogers ${ }^{\ddagger}$ \\ ${ }^{\star}$ Faculty of Engineering and the Environment \\ University of Southampton, Southampton SO17 1BJ, UK \\ ${ }^{\dagger} \mathrm{ABB}$ Corporate Research \\ Zurich, Switzerland \\ ${ }^{+}$Centro Italiano Ricerche Aerospaziali ScpA \\ $\ddagger$ Electronics and Computer Science \\ University of Southampton, Southampton SO17 1BJ, UK
}

* Author for correspondence.

ort@soton.ac.uk

fax: +442380593058

Running title: Control of flow with trapped vortices

Keywords: Vortex cells, flow control, separation control, controller design.

\begin{abstract}
The results in this paper arise from an investigation into control strategies designed to trap a vortex in a cavity such that the flow remains attached with no large scale vortex shedding. Progress in this general area is required to advance the development of thick winged aircraft where, for example, such wings could be used to store more fuel. A discrete vortex computational dynamics model of the flow is used to develop simple but effective control strategies. In this paper, the use of constant suction or a PI controller to stabilize the flow, followed by oscillatory open loop suction to maintain stability with a significantly reduced effort is investigated. To support the simulation studies, results from wind tunnel experiments are given.
\end{abstract}




\section{Introduction}

Wings on modern aircraft have a characteristic long thin streamlined shape, driven by the need to maintain a high lift to drag ratio. However, from a structural viewpoint, thicker wings would be preferable as they have a greater load bearing capacity. With the increase in size of transport aircraft, the balance between the structural and aerodynamic requirements shifts in favor of thicker wings. Hence there is much current interest in the design of thick-wing or blended wing-body aircraft. Thick wings could also be beneficial in certain types of small aircraft. An example is the High Altitude Long Endurance (HALE) aircraft where the wings could be used for fuel storage and hence a larger volume is highly desirable.

The difficulty with this idea is that flow past a thick body is highly likely to separate; as the body narrows towards its trailing edge, the flow undergoes a rapid deceleration, and if the body is sufficiently thick, without intervention it will leave the surface of the body leading to a large region of recirculating flow and large scale eddy (vortex) shedding. The result is a drop in lift, a large increase in drag, and fluctuations in the loads (lift and drag) on the body. In conventional aircraft, this is what occurs when an aircraft stalls, but for such aircraft stall can be avoided by maintaining the speed of the aircraft. In contrast, for thick bodies the flow will separate at all flow speeds relevant to aircraft. Hence one problem that must be addressed in order to make thick-winged aircraft realizable is how to suppress separation in a efficient manner so that aerodynamic performance is maintained.

One feasible way of doing suppressing this separation is to place a cavity on the surface of the body around the point that separation would naturally occur. When separation occurs the velocity of the flow along the body near the surface drops to zero, with reversed flow adjacent to the wall downstream of the separation point. With a cavity, the velocity of the flow across the mouth of the cavity would be non-zero, moving the separation point downstream if not preventing separation altogether. In effect, the flow across the mouth of the cavity would act as a moving wall so that the flow speed in this region does not go to zero. This situation is illustrated in Figure 1.

The idea of trapping a vortex is not new. For example, it appears in [1]. The first known successful use in practice in a flight experiments was by Kasper [2], although attempts to reproduce Kasper's results in wind tunnel test were not successful due to vortex shedding [3]. However, a $1.3 \mathrm{~m}$ wingspan model aircraft which utilizes vortex cells has been flown successfully [4].

The major problem in employing vortex cells in practice is that the flow is inherently unstable, and the instability leads to a strong non-linear interaction between the flow in the cavity and the external flow past the cavity, resulting in large scale vortex shedding, i.e. exactly the condition that the cavity was meant to prevent. Hence the key problem which must be addressed in order to make this technique for controlling separation usable in practice is to find an efficient means of suppressing the large scale vortex shedding so that the vortex is trapped inside the cavity and that external flow passes the mouth of the cavity as indicated in Figure 1.

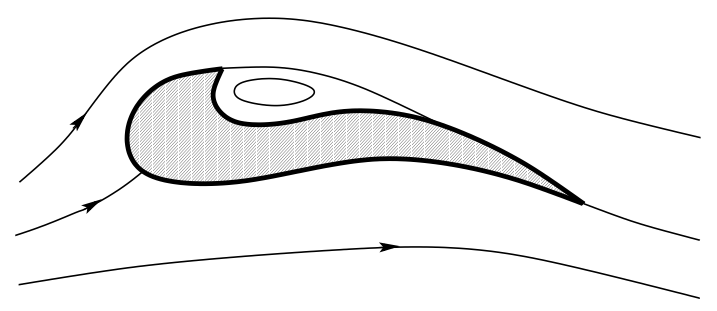

Figure 1: Sketch of a flow with a trapped vortex.

The flow can be stabilized by means of suction within the cavity, as discussed below. In practice this 
needs to be done in an efficient manner, i.e. so that the energy expended in stabilizing the flow is such that there is a net energy gain. This requires not simply a gain over the device with no control but more efficiently than if control was used on an airfoil of the same shape but with no cavity.

The major aim of this paper is to develop an efficient control strategy for the flow past an airfoil with a cavity using surface suction as the actuation. Suction is commonly used for flow control purposes as it is well established that relatively small amounts of suction can have a significant effect on the flow, for example, in controlling boundary layer transition (see e.g. [5, 6]). Further, it is possible to construct suction systems of the kind considered here (e.g. [5]).

In general terms the aim of any flow control strategy would be to minimize the total power consumed by the system. However, this would be a complicated function depending on not just the loads on the body (in particular the drag), but on other factors such as the power consumed by the actuation (the suction system), the increase in weight, and the cost of installing and maintaining the control system. Thus the cost function for a fully working system will depend of the exact configuration of the system. Here we require a simpler measure. We will aim to minimize the mass flux of the suction required. This is easily measured and is a realistic measure as in general power as the cube of velocity and hence as the cube of the mass flux for an incompressible flow.

In addition to the primary aim of stabilizing the flow, which the ultimate intention is to apply the control in a physical situation, an important secondary aim is to develop the simplest possible control strategy that can achieve the desired results. Note that stabilizing the flow, we are referring explicitly to suppressing large scale eddy shedding/separation, not suppressing all unsteadiness in the flow. In particular, even if the flow is fully attached, there will still be small scale fluctuations due to the fact that the flow in the boundary layer near the wall will inevitably be turbulent at the flow rate considered.

The basic problem is described in the next section, followed by the numerical means used to model the flow. We then consider simple but effective methods for stabilizing the flow and reducing the control cost by active control. Although the major aim of this paper is to develop feasible control strategies for trapping vortices using a modelling/theoretical approach, a short series of experiments was conducted using a wind tunnel model of the test configuration. Relevant aspects from these experiments, performed at Centro Italiano Ricerche Aerospaziali (CIRA), are considered. Finally, some conclusions are drawn.

\section{The Basic Problem}

The experimental configuration used in this work is an unusual one in that the airfoil is not mounted in the center of the wind tunnel but on the lower wall as shown in Figure 2. Note that we are not investigating the flow past an airfoil as such, but flow past a cavity designed to trap a vortex provided the flow can be stabilized. This particular configuration was adopted for a number of reasons. First, the model is large compared to the size of the wind tunnel $-0.35 \mathrm{~m}$ chord in a tunnel with a $0.3 \times 0.305 \times 0.6$ $\mathrm{m}(\mathrm{HxWxL})$ - working section and placing it in the center of the tunnel would cause unacceptably large blockage effects. Second, placing the model on the wall provides access for the suction system used for actuation. Third, it also provides access for the PIV (Particle Image Velocimetry) system used for optical measurement of the flow field. Fourth, the pressure gradient over the cavity can be altered by changing the angle of attack of the airfoil. Thus, this design satisfies our requirements, and allows a relatively large cavity compared to the size of the working section of the wind tunnel. Also, suction was applied on the lower wall of the tunnel upstream of the model in order to suppress the large scale, unsteady, separation that would otherwise occur ahead of the model, providing an undesirable disturbance to the oncoming flow.

Details of the cavity are shown in Figure 3. This shows the position and size of the suction slots and the position of the pressure taps that can be used to assess the mean state of the flow. The pressure taps measure only the mean value of the surface pressure and they do not provide any time dependent 


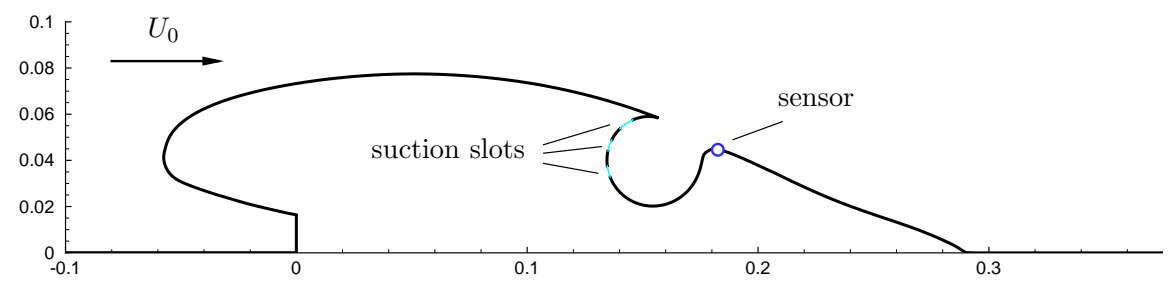

Figure 2: The airfoil mounted on the wall of the wind tunnel. Also shown are the positions of the three suction slots used for control and the position of a sensor which can be used for feedback control. Lengths are in meters.

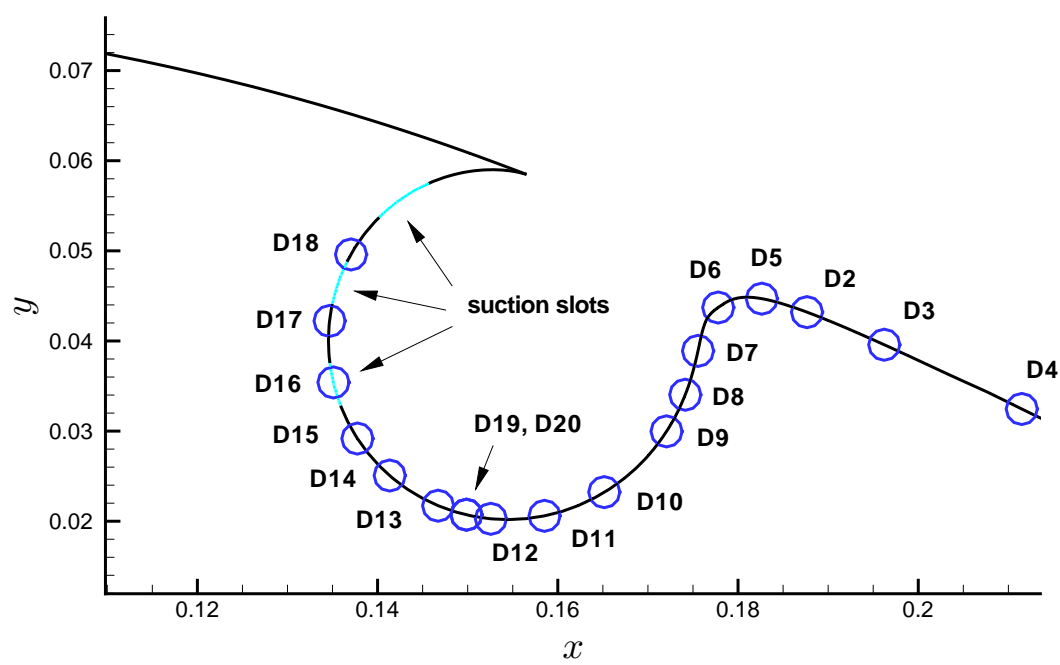

Figure 3: Details of the cavity. D1-D20 are pressure taps in CIRA experiments. D1 is situated upstream of the cavity tip. D1-D18 are located along the midpoint of the airfoil. D19 and D20 are in the different spanwise locations. Lengths are in meters.

information on the fluctuations, including those due to the large scale eddy shedding of most interest here. The suction slots are placed on the upstream side of the cavity because this is the most appropriate place for them in the wind tunnel experiments and because this is where they would be placed on cavities in the wings of a HALE aircraft, the target vehicle. The suction slots are connected to a pump through a system of valves and flow meters which can be used to adjust and measure the flow rate for each of the slots.

The suction slots are connected to a pump through a system of valves and flow meters which can be used to adjust and measure the flow rate for each of the slots.

\section{Modeling the Flow}

Given the restrictions on the amount and type of data that can be collected from the test bed, plus the fact that the wind tunnel is available only for short, infrequent periods of time, a reliable numerical 
model of the flow is required in order to develop a control strategy. Also, as repeated runs with different operational parameters (angles of attack for the model, wind tunnel speeds, suction flow rates) and possible control schemes are required, it is not possible to use a full three dimensional Navier-Stokes solver for this task (a single run would take $O\left(10^{4}-10^{5}\right)$ processor hours). However, the model is mounted normal to the flow in the wind tunnel, and away from the walls, the flow would be expected to be essentially two-dimensional in nature (this was confirmed in the experiments performed at CIRA, using tufts on the surface of the model and PIV data from slices across its span). Hence a two-dimensional incompressible Navier-Stokes solver was used. The code uses a Discrete Vortex Method (DVM). DVM's are Lagrangian methods which simulate the flow by tracking the motion of the vorticity field which is partitioned into a finite number of particles (vortex blobs). Much information on DVM's in general can be found in [7]. The code used here was based on that described in [8], but with the viscous part of the calculation based on a vorticity redistribution method $[9,10]$.

The Navier-Stokes equations governing two-dimensional incompressible flow can be written in vorticity form as

$$
\frac{D \omega}{D t}=\frac{\partial \omega}{\partial t}+v_{x} \frac{\partial \omega}{\partial x}+v_{y} \frac{\partial \omega}{\partial y}=\frac{1}{R e} \nabla^{2} \omega
$$

where $\omega=\partial v_{y} / \partial x-\partial v_{x} / \partial v_{y}$ is the vorticity, and $D / D t=\partial / \partial t+v_{x} \partial / \partial x+v_{y} \partial / \partial y$ is the material derivative, i.e. the rate of change with time of a material quantity convected with the flow. $\nabla^{2}$ is the Laplace operator, and the Reynolds number $R e$ is given by $U_{0} L / \nu$ where $\nu$ is the kinematic viscosity of the fluid, $U_{0}$ is the free stream (reference) velocity in the wind tunnel, and $L$ is a reference length, taken as $1 \mathrm{~m}$. Time $t$ is non-dimensionality using the reference time $L / U_{0}$. An operator splitting method is used, with inviscid and viscous substeps, satisfying

$$
\frac{D \omega}{D t}=0
$$

and

$$
\frac{\partial \omega}{\partial t}=\frac{1}{R e} \nabla^{2} \omega
$$

respectively. The equation for the inviscid sub step represents the fact that for two-dimensional inviscid flow vorticity is convected by the flow [11].

The vorticity field is represented by $N_{v}$ discrete vortex blobs so that

$$
\omega=\sum_{j=1}^{N_{v}} \Gamma_{j} \gamma\left(\left|\mathbf{x}-\mathbf{x}_{j}\right|\right)
$$

where $\Gamma_{j}$ is the strength of vortex $j$, which is located at $\mathbf{x}=\mathbf{x}_{j}$ and $\gamma(\eta), \eta=\left|\mathbf{x}-\mathbf{x}_{j}\right|$, is the (axisymmetric) distribution of vorticity in a blob. This generates a velocity field

$$
\left(u_{v}, v_{v}\right)=\sum_{j=1}^{N} \Gamma_{j} \frac{\left(-y+y_{j}, x-x_{j}\right)}{\left|\mathbf{x}-\mathbf{x}_{j}\right|^{2}} F\left(\left|\mathbf{x}-\mathbf{x}_{j}\right|\right)
$$

where

$$
F(\eta)=\int_{0}^{\eta} \gamma(s) s d s
$$

Here, the standard Gaussian distribution with

$$
\gamma(\eta)=\frac{1}{\pi \sigma^{2}} e^{-\eta^{2} / \sigma^{2}}, \quad F(\eta)=\frac{1}{2 \pi}\left[1-e^{-\eta^{2} / \sigma^{2}}\right]
$$

is used, where $\sigma$ is a measure of the core size of the vortex. 
Numerically, the vorticity field is updated at each time step by moving individual vortex blobs as the solution of

$$
\frac{d \mathbf{x}_{j}}{d t}=\mathbf{v}\left(\mathbf{x}_{j}, t\right)
$$

where $\mathbf{j}_{v}$ is the position of the core of the $j$ th vortex blob (the inviscid step), and by transferring circulation between vortex blobs using the redistribution of Shankar and van Dommelen [9] (the viscous substep). Here, as in [8], a second order Runge-Kutta method is used to move the vortices.

A panel method is used to satisfy the boundary conditions of the walls of the tunnel. Both source and vortex panels are used in order to set the tangential velocity to zero, but allow a non-zero normal velocity at the positions of the suction panels, with zero normal velocity elsewhere. Details of vortex and source panels and the velocity fields they generate can be found in many standard texts (e.g. [12]).

The final velocity field consists of three components, the incoming free stream velocity $\left(U_{0}\right)$, the contribution from the panel at the walls, and the velocity generated by the vortex blobs (5), the sum of which is used to move the vortices at each time step (8).

The vorticity redistribution method in [9] is formulated for laminar flow, with a constant viscosity/Reynolds number. However, it is easily modified for turbulent flow by the use of a turbulent viscosity $\mu_{t}$, replacing $1 / R e$ in $(3)$ by $\left(1+\mu_{t}\right) / R e$. This was done using a second-order velocity structure function model $[13,14]$, as this class of model can be used with an irregular distribution of grid points as occurs with the Lagrangian DVM model. Calculations were performed with and without the turbulence model. In terms for the phenomena of interest here, the ability of the control to stabilize the flow by suppressing the large scale vortex shedding of the flow, the results were essentially the same with or without the turbulence model.

In this work, 1202 vortex and source panels where used, 1001 on the lower wall, and 201 on the upper wall. Vortices were shed from the lower wall at each time step, following the scheme in [8]. The nondimensional time step used was 0.01 , although tests were performed with a smaller time step (0.005) to ensure that the results were essentially independent of the time step.

Figure 4 shows typical instantaneous streamlines in the region of the cavity from a simulation of the flow with the free stream velocity of $U_{0}=30 \mathrm{~m} / \mathrm{s}$ (a Reynolds number of $7.3 \times 10^{5}$ ) and the airfoil at $7^{\circ}$ angle of attack $\left(7^{\circ} \mathrm{AoA}\right)$. Figure 5 shows the corresponding vorticity distribution. Both of these plots clearly show the large scale vortex shedding that we wish to suppress.

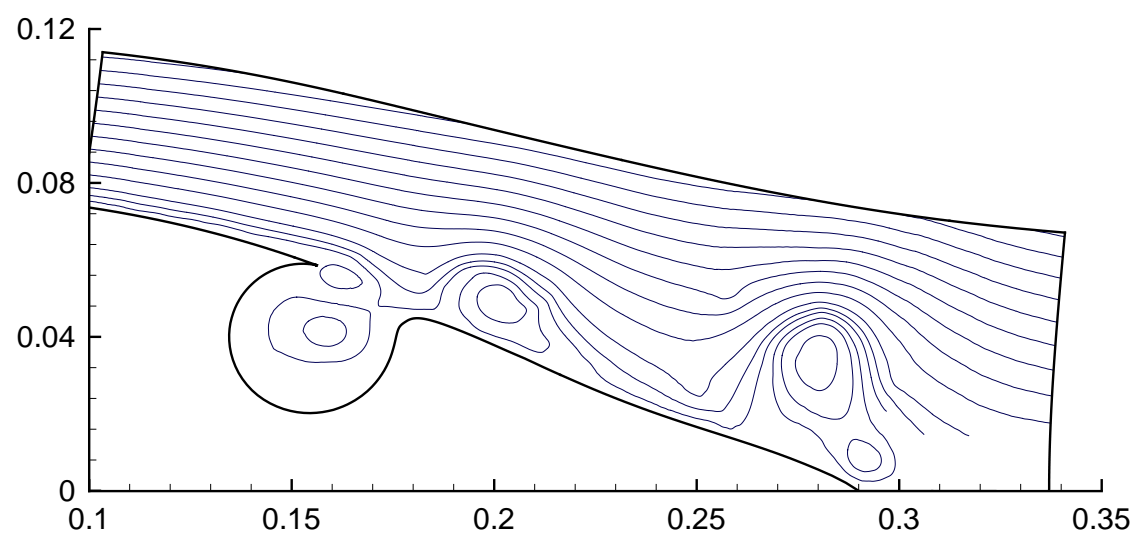

Figure 4: Instantaneous streamlines for the flow with no suction.

Figures 6 and 7 show the flow at the same flow condition with a $4 \%$ suction flow rate, i.e. with $u_{s}=0.04 U_{0}$ where $u_{s}$ is the suction velocity normal to the surface on the suction panels. The same suction velocity 


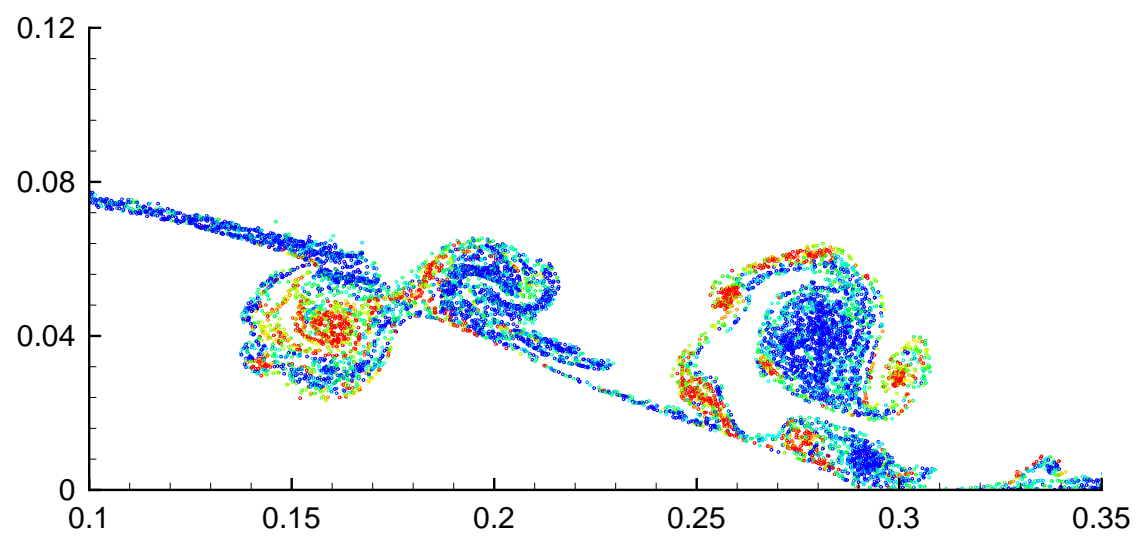

Figure 5: Instantaneous vorticity field for flow with no suction.

is used on each panel. The large scale vortex shedding has been suppressed by the suction.

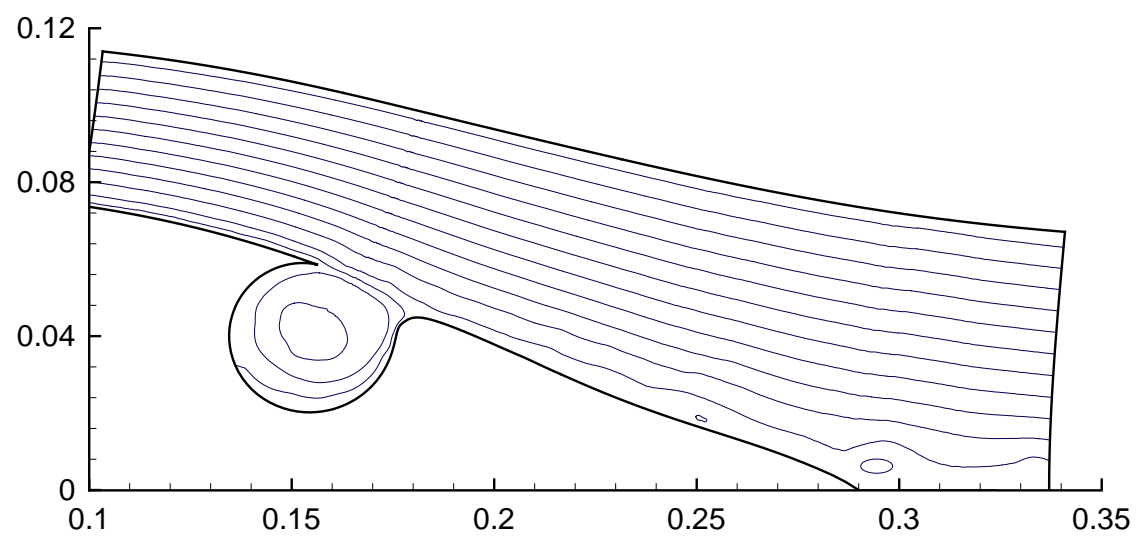

Figure 6: Instantaneous streamlines for the flow with $C_{q}=u_{s} / U_{0}=0.04$.

A similar set of calculations were performed using the same configuration but no cavity. It was found that more than twice as much suction, in terms of the mass flow rate, was required in order to force the boundary layer to remain attached. The same conclusion was reached in [15] using a completely different numerical approach. Hence, the use of cavity should greatly improve the efficiency of the system in terms of the energy required to stabilize the flow.

\section{Stabilizing the Flow}

As illustrated in Figures 6 and 7, it is possible to stabilize the flow by applying constant suction, provided the angle of attack is not so large that separation occurs upstream of the cavity. If the critical constant suction rate where known for all possible operating conditions (AoA and free stream flow rate) for a particular configuration (airfoil shape, cavity shape and position), then the suction could be set at a rate greater than the critical value providing that the operating conditions (and changes in them) could be 


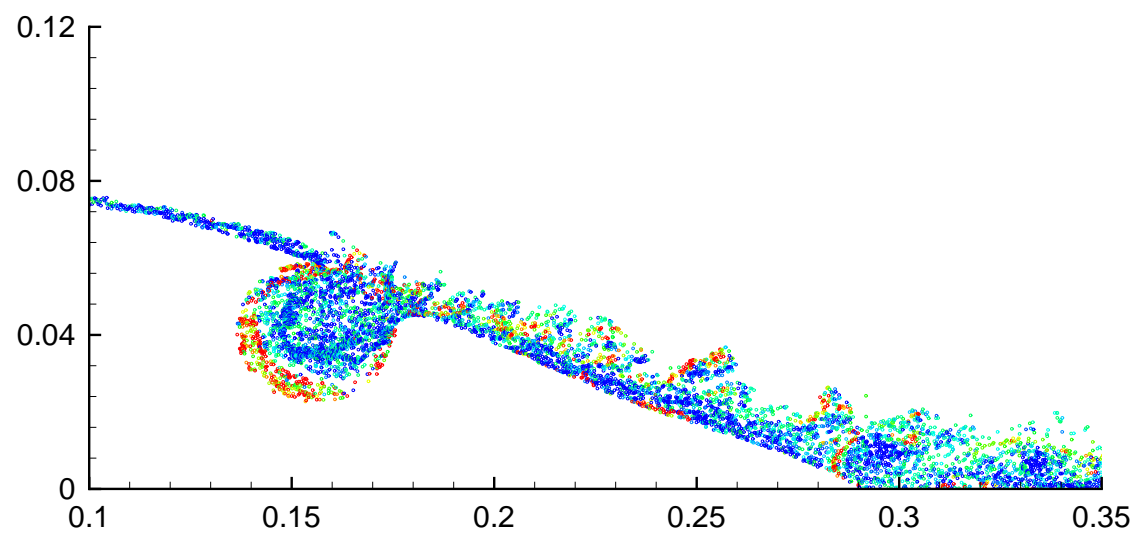

Figure 7: Instantaneous vorticity field for flow with $C_{q}=u_{s} / U_{0}=0.04$.

determined. However, an active scheme which can determine the minimum suction required to stabilize the flow would be preferable.

In order to implement such a scheme we need some measure of the state of the flow to use as an input. This should be based on a quantity that can be measured in an experimental situation. In practice there are two quantities which could be used, the shear stress or pressure at the surface of the body, which are, respectively, the tangential and normal forces exerted by the fluid on the surface of the body. From a fluids viewpoint, the shear stress would be preferable as it relates most closely to the phenomenon we wish to control, and it is used for sensing in many theoretical studies of flow control. However, it is much more difficult to measure the wall shear stress than the pressure, and the latter is the usual quantity measured in experiments. Hence we will use a quantity based on the pressure.

The DVM uses source and vortex panels to enforce the boundary conditions for the velocity at the surface (see [8] for details). The strength of the vortex panels is proportional to the pressure gradient along the surface, and is therefore the obvious quantity to use. Experimentally, this quantity could be obtained by placing two time-accurate pressure sensors a suitable distance apart on the surface of the body.

Figure 8 shows the mean values $\left\langle\lambda_{i}\right\rangle$ and standard deviation $\sigma_{i}$ for (a scaled value of) the strength of the vortex panels $\left(\lambda_{i}\right)$ as a function of constant suction flow rate $\left(C_{q}\right)$ at a number of locations corresponding to the sensor locations shown in Figure 3 . The model is at $7^{\circ}$ AoA with $U_{0}=30 \mathrm{~m} / \mathrm{s}$. Not surprisingly, sensor D1, which is upstream of the cavity, shows no effect from the actuation. For sensor D5, which is near the downstream lip of the cavity, there is a large drop in both the mean value and variance of the signal when $C_{q}=0.04$, For sensor D6, which is also near the lip, the mean increases but the variance drops at this value of $C_{q}$. The decrease in $\sigma_{i}$ directly reflects the suppression of the large scale vortex shedding; large intermittent structures in the flow will have a direct effect of the pressure distribution on the surface and their suppression will cause a decrease in the fluctuation of the pointwise values of the pressure and the pressure gradient. Figures 6 and 7 confirm that the vortex shedding has been suppressed with $C_{q}=0.04$.

For sensor D18 (and for other sensors on the upstream wall of the cavity) the mean value of the sensor reading is not affected by the actuation but the variance increases with $C_{q}$. This increase in $\sigma$ is probably due the entrainment by the suction of unsteady flow from the boundary layer upstream of the cavity into the cavity.

Figure 9 shows time traces of the signal ( $\lambda$ against time in expressed in non-dimensional terms using 
(a) sensor D5

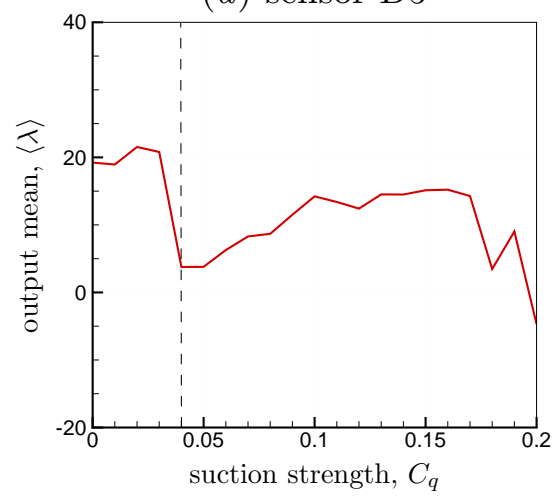

(c) sensor D6

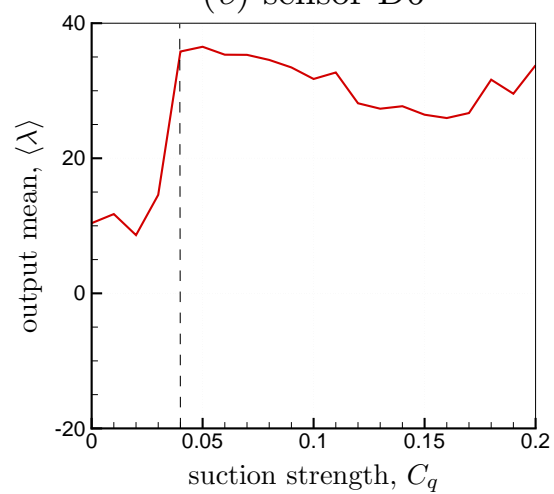

(e) sensor D18

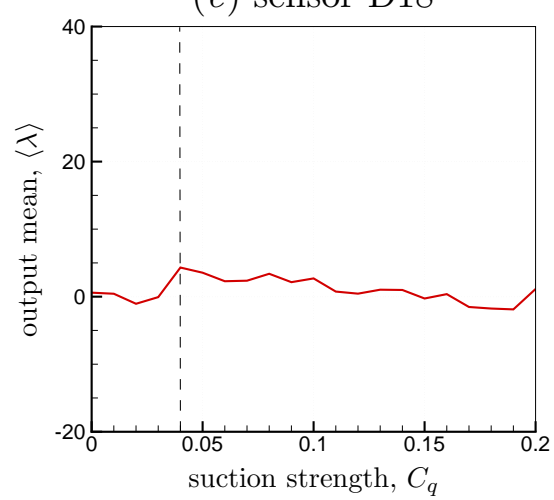

(g) sensor D1

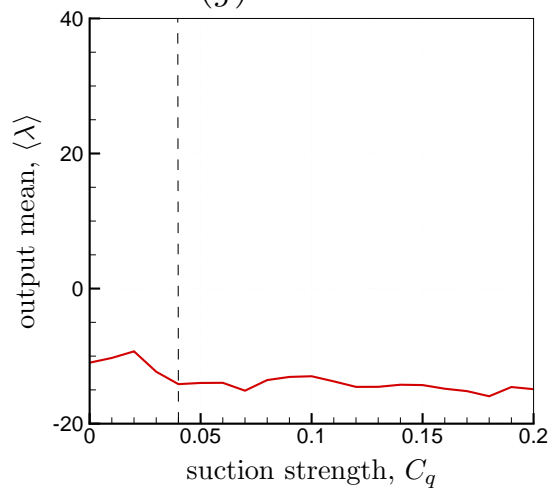

(b) sensor D5

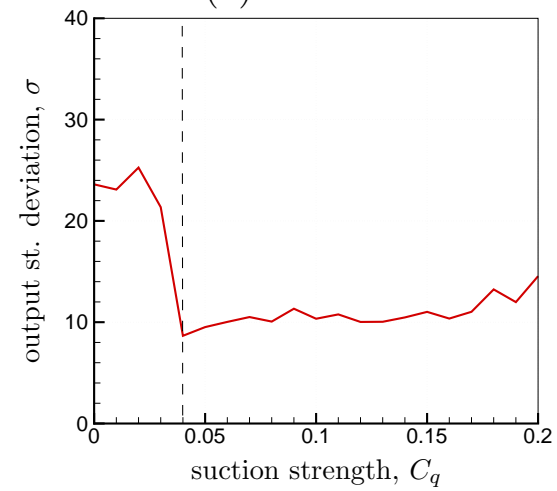

(d) sensor D6

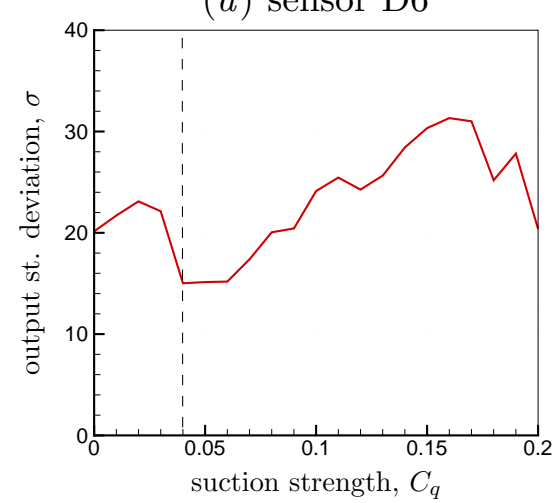

$(f)$ sensor D18

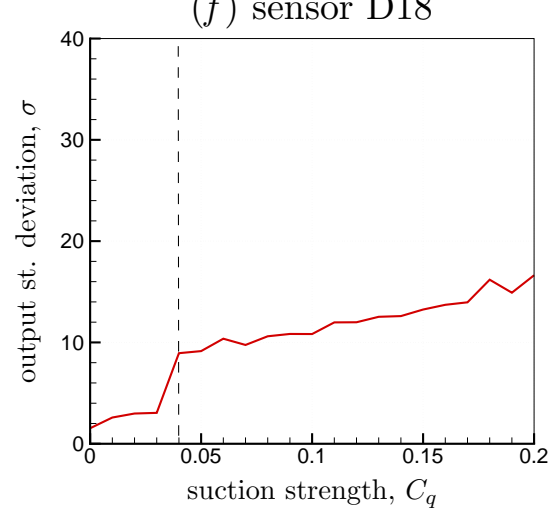

(h) sensor D1

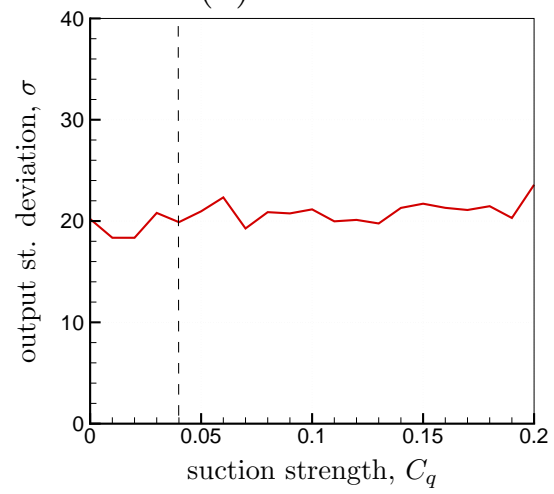

Figure 8: Mean values and standard deviations of the output signal $\lambda$ vs. suction strength at various sensors. 
(a) D5, no suction

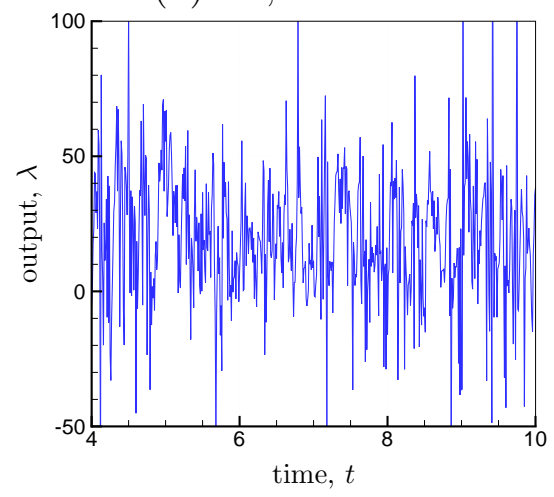

(c) D6, no suction

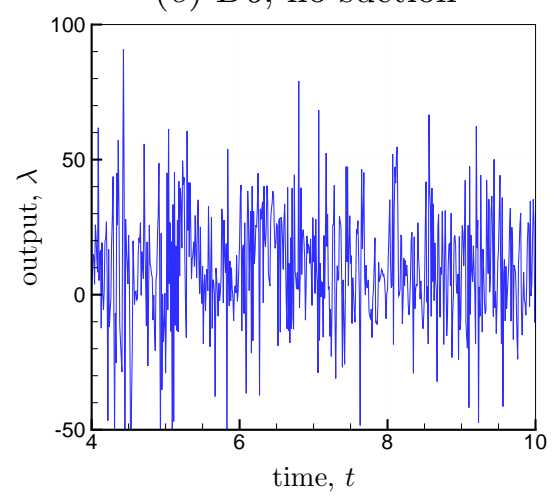

(e) D18, no suction

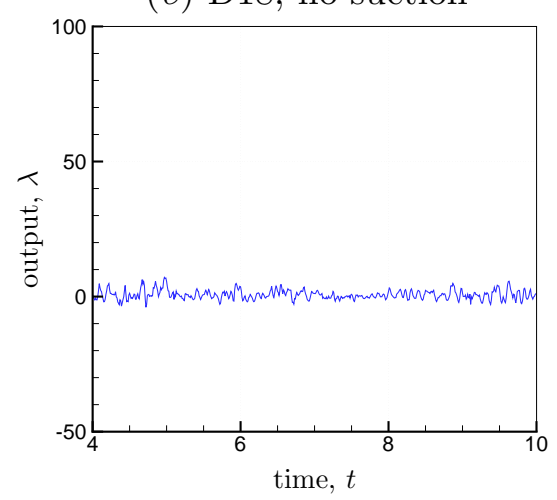

(g) D4, no suction

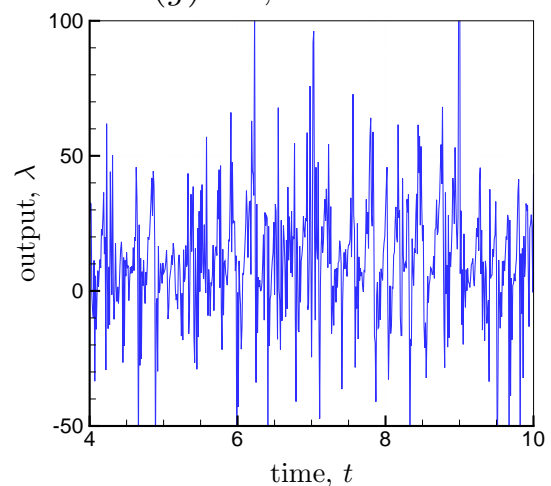

(b) $\mathrm{D} 5, S=0.04$

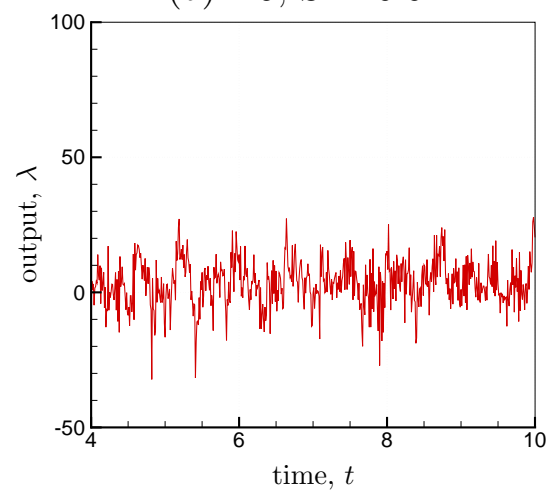

(d) $\mathrm{D} 6, S=0.04$

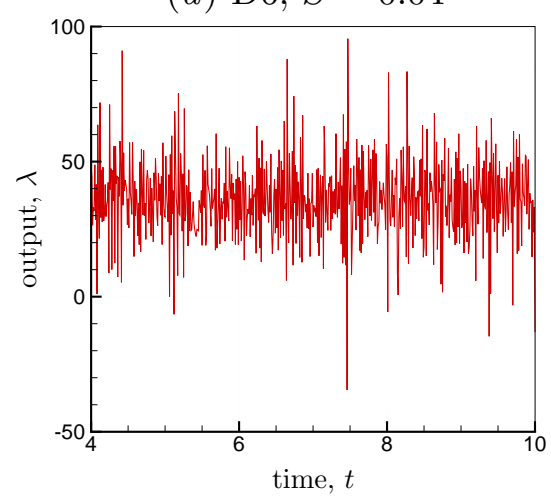

(f) $\mathrm{D} 18, S=0.04$

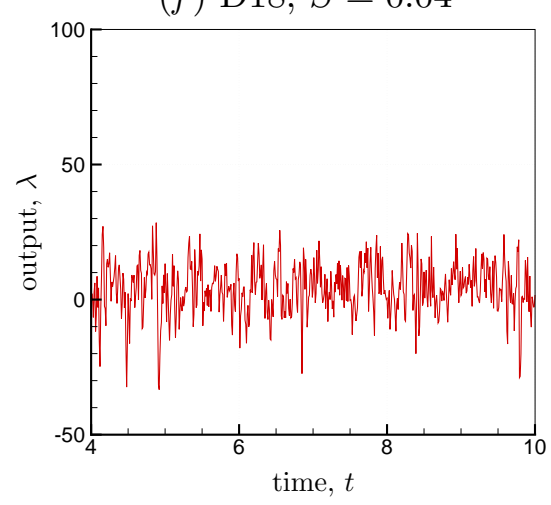

(h) $\mathrm{D} 4, S=0.04$

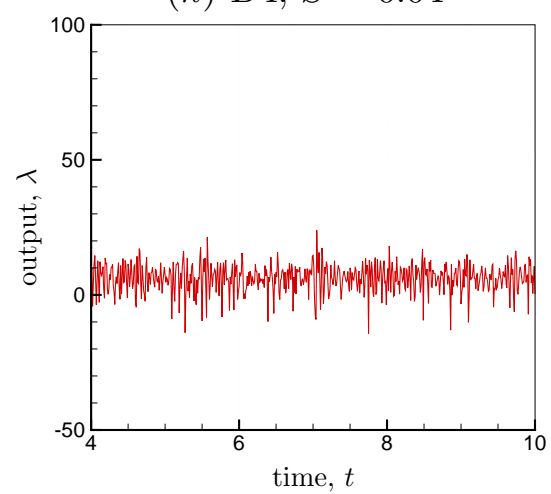

Figure 9: Time histories of the output signal $\lambda$ for various sensors with and without suction. 
(a)

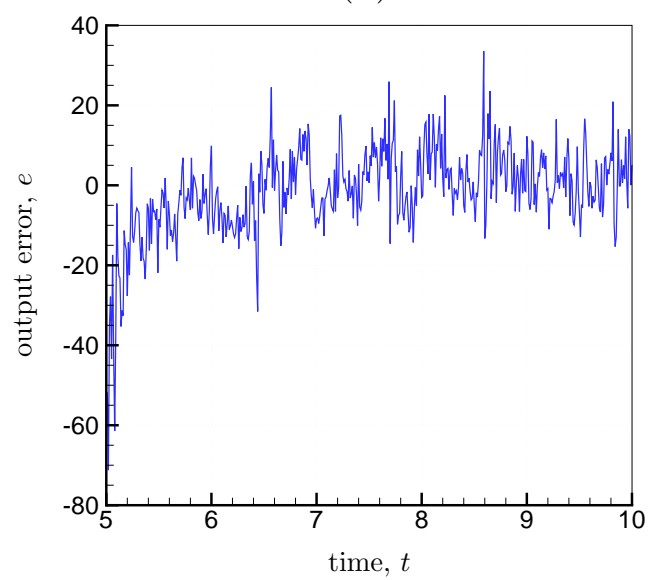

(b)

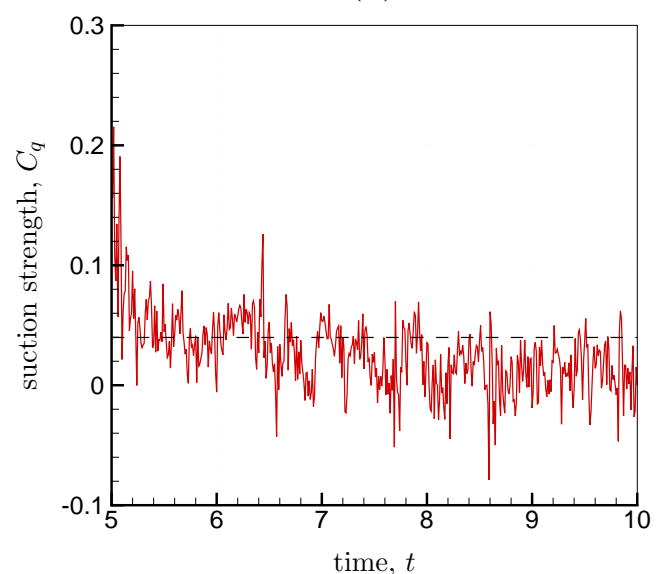

Figure 10: Time histories of $(a)$ output error $e$, and $(b)$ suction strength $C_{q}$ for PI control with $K_{p}=-.003, K_{i}=-0.002$ and $C_{q 0}=0.15$. The PI controller is activated at $t=5$ with zero suction for $t<5$.

$L / U_{0}$ where $L$ is a the reference length of 1 meter $)$ for the case with no suction $\left(C_{q}=0\right)$ and that with the critical value which suppresses the vortex shedding $\left(C_{q}=0.04\right)$. Sensors D5 and D6 shows the decrease in the magnitude of the fluctuations with suction and D18 an increase, as would be expected from Figure 8. Sensor D4, which is downstream of the cavity, also shows a decrease, again reflecting the suppression of the vortex shedding.

Sufficiently large suction will suppress the vortex shedding. However, in Figures 8 and 9 there is still a significant unsteady component in the signals from the sensors, due to the remaining small relatively small scale fluctuations in the flow. Here we are not interested in suppressing these fluctuations (this would require a large amount of energy), only the larger scale disturbances.

It is clear from Figure 8 that the mean value of the signal for sensor D5 or D6 can be used as a measure of the state of the flow, in particular to determine whether it has been stabilized. We have used the signal from D5, defining $e(t)=\lambda_{0}-\lambda_{5}(t)$ as the output error, where $\lambda_{0}=3.8$ is the mean value of the scaled pressure gradient for constant suction with $C_{q}=0.04$. We assume that all the suction panels have the same adjustable suction flow rate so we have a single-input single-output (SISO) system. A Proportional plus Integral(PI) controller was designed, with the control law

$$
C_{q}(t)=K_{p} e(t)+K_{i} \int_{t_{0}}^{t} e(t) d t+C_{q 0}
$$

where $t_{0}$ is the time the control is turned on, $K_{p}$ and $K_{i}$ the proportional and integral gains, respectively and $C_{q 0} \geq 0$ is constant. The time $t$ is non-dimensional, scaled by the reference time $L / U_{0}$. The sample time for the control here and below was the same as the time step for the simulation, i.e. $0.01 \mathrm{~L} / U_{0}$.

The gains were tuned using the Ziegler-Nichols auto-tuning rules [16], resulting in $K_{p}=-0.003$ and $K_{i}=-0.002$. Figure 10 shows time histories of the output error and the suction strength with the control turned on at $t=5$ with $C_{q 0}=0.15$. The mean output error rapidly tends to zero and the suction strength settles on a value close to the critical value determined earlier using constant suction. Clearly the PI control has stabilized the flow with a reasonable if not optimum value of the suction.

The constant $C_{q 0}$ is included in (9) so that the initial suction velocity can be forced to be positive regardless of the value of $e$ at $t=t_{0}$. In general, suction stabilizes flow while blowing $\left(C_{q}<0\right)$, destabilizes flow. However, we would expect the control to eventually stabilize the flow with initial 

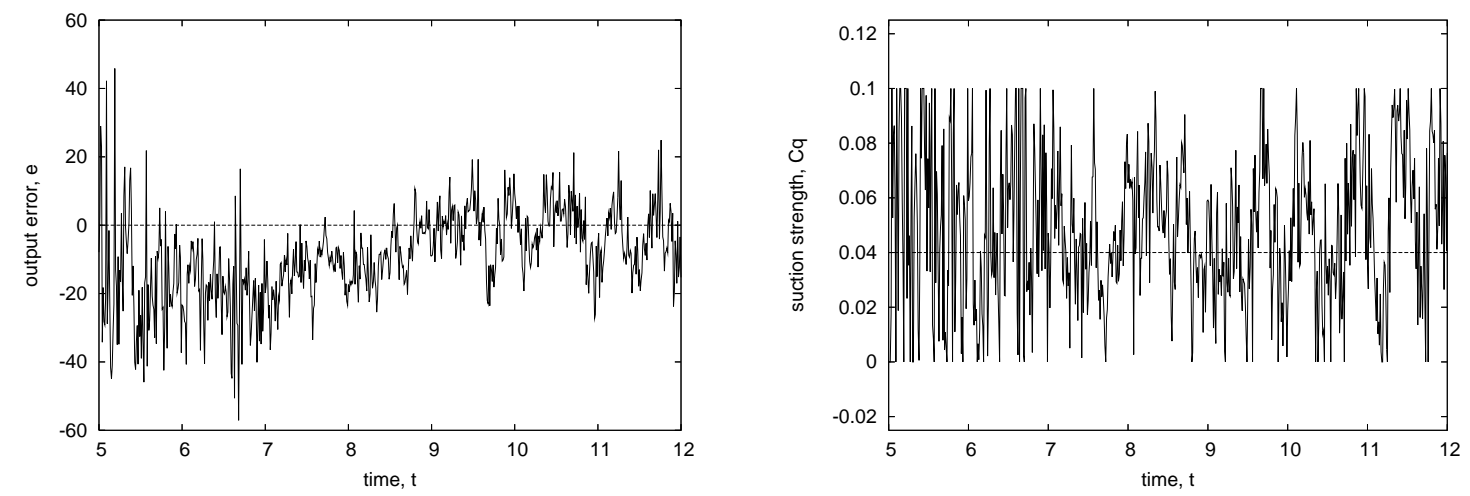

Figure 11: Time histories of $(a)$ output error $e$, and $(b)$ suction strength $C_{q}$ for PI control with $K_{p}=-.003, K_{i}=-0.002$ and $C_{q 0}=0.15$ with the actuation limited to $0 \leq C_{q} \leq 0.1$. The PI controller is activated at $t=5$ with zero suction for $t<5$.

blowing if the gains have been chosen appropriately. In fact, these was seen for the case used for Figure 10 with $C_{q 0}$ is set to zero. There was strong blowing when the control is turned on at $t=5$, but the flow did stabilize, although it took until $t \approx 10$ for this to occur, rather than $t \approx 5.5$ as in Figure 10 .

Figure 10 shows a significant amount of blowing $\left(C_{q}<0\right)$ as well as high levels of actuation. In practice there would be limits on the suction velocity, while a system with both suction and blowing would be much harder to build than one with suction (or blowing) only. Accordingly, the actuation was restricted to suction $\left(C_{q} \geq 0\right)$ with a maximum possible suction velocity of $10 \%$ of the freestream velocity $\left(C_{q} \leq 0.1\right)$. The output error and the suction flow rate for this case are shown in Figure 11. Again, the control is successful, but it takes until $t \approx 9$ for the flow to be stabilized. For $t=9$ until $t=12$, the mean values of the error and the suction flow rate are $-1.75(\lambda=4.55)$ and 0.049 respectively. Thus, there is a higher suction flow rate with this control than that identified as the critical value for constant suction (Figure 8(a)). However, for Figures 8 and 9 the suction was turned on throughout the calculation, i.e. from the impulsive start at $t=0$. If instead there is initially no suction so that an unstable flow with large scale shedding develops, as for the calculation with the PI control, then applying constant suction with $C_{q}=0.04$ for $t>5$ did not stabilize the flow for a calculation running until $t=24$. In contrast, applying suction with $C_{q}=0.05$ at $t>5$ quickly stabilized the flow (by $t=7$ ). Thus $C_{q}=0.04$ can but does not necessarily stabilize the flow, while $C_{q}=0.05$ does even when starting from a highly disturbed flow. A mean value of $C_{q} \approx 0.05$ for the PI control with limited suction is therefore a realistic outcome.

The difference in critical suction flow rate for the case with non-zero suction at the start of the run and suction applied at $t=5$ arises from the fact that in general it takes more energy to re-stabilize a flow with large scale instabilities than to maintain the stability of a flow in an essentially undisturbed state.

From Figure 8a we see that there is more than one constant suction value that can produce an average output of the target value 3.8. In fact, by limiting the actuation to suction only $\left(C_{q}>0\right)$, but with no upper limit on the value of suction, the control converged onto the target value but with $C_{q}$ varying around 0.20, consistent with the data shown in Figure 8a.

\section{Open-Loop Control}

In the previous section a PI controller that can stabilize the flow has been designed. However, implementing this controller would be difficult because of the rapid fluctuations in the actuation. Thus, a 


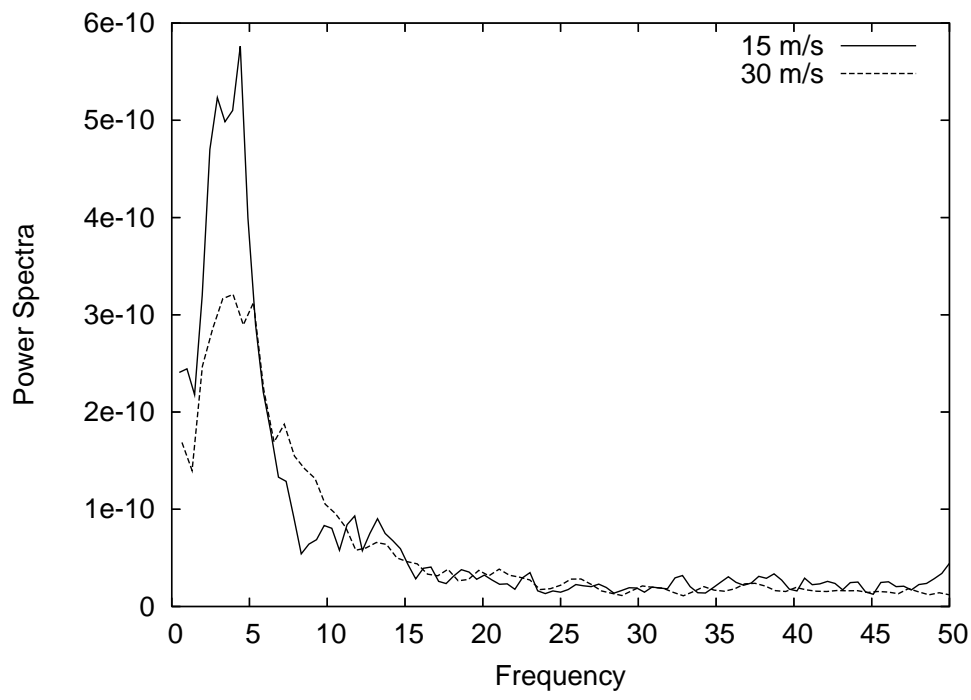

Figure 12: Non-dimensional power spectra for the output from sensor D5. The frequency is in units of $U_{0} / L$.

simpler, more easily implemented control would be desirable. Here we demonstrate how the efficiency of the control may be significantly improved by applying simple open loop unsteady actuation. Commonly, in flow control, pulsed/oscillatory actuation is more efficient that steady state actuation. Hence we consider oscillatory suction. Figure 12 shows the non-dimensional power spectra for the output for sensor D5 with $U_{0}=15$ and $30 \mathrm{~m} / \mathrm{s}$. For both speeds, the spectra is concentrated around a frequency of $4 U_{0} / L\left(60 \mathrm{~Hz}\right.$ for $U_{0}=15 \mathrm{~m} / \mathrm{s}$ and $120 \mathrm{~Hz}$ for $\left.U_{0}=30 \mathrm{~m} / \mathrm{s}\right)$. From examination of the PIV data (see $\S 7$ below), the shedding frequency, and general behavior of the flow, in the simulations is consistent with that seen in the experiments at similar conditions.

Oscillatory suction in the form

$$
C_{q}=A_{0}+A_{1}\left[\cos \frac{2 \pi}{T}\left(t-t_{0}\right)-1\right]
$$

where $A_{0}$ and $A_{1}$ are positive constants, the actuation is switched on at $t=t_{0}$ with (non-dimensional) period T. $C_{q}$ varies between $A_{1}-2 A_{0}$ and $A_{0}$ with a mean of $A_{0}-A_{1}$ starting from $A_{0}$ at $t=t_{0}$. We assume that the system is for suction only so that the maximum possible value of $A_{1}$ is $A_{0} / 2$.

Open loop actuation of the form (10) has a major effect on the pointwise surface pressure gradient. In particular, when holding the range of the actuation constant (i.e. with fixed values of $A_{0}$ and $A_{1}$ ), the value of the period $(T)$ has a strong effect on the mean value of the surface pressure gradient at the sensor position D5 as used above as the input for the PI control, even when the large scale eddy shedding has been suppressed. Hence, here we use direct observation of the flow field to determine whether the flow has been stabilized. In particular animations of the vorticity field were used as this quantity is the primary variable used in the numerical method and the presence of large scale vortices is immediately apparent from the vorticity distribution, as can be seen in Figure 5.

A wide range of values of parameters of the actuation was investigated with the model at the design conditions of $7^{\circ} \mathrm{AoA}$ and $R e=2.1 \times 10^{6}\left(U_{0}=30 \mathrm{~m} / \mathrm{s}\right)$. The primary aim was to minimis the mean value of the actuation while stabilizing the flow. It was found that when starting from a flow with constant suction with $C_{q}=0.03$, which is unstable, the flow could be stabilized if the suction was varied between zero and 0.03 (i.e. with $A_{0}=0.03$ and $A_{1}=0.015$ ).

Figure 13 shows the vorticity distribution for the flow at $t=5$ when the control is turned on and the flow 
at $t=6$ for actuation with $T=0.1$. The large scale eddies which can be seen in the flow with constant suction with $C_{q}=0.03$, which is below the critical value of 0.05 , have been suppressed entirely by the oscillatory suction, with a very thin attached boundary layer. Although Figure 13 shows the vorticity at a specific time, it is representative of the flow once the unsteady actuation has taken affect. Given the scaling of power as velocity squared, this oscillatory actuation has a power requirement more than an order of magnitude less than that with constant suction with $C_{q}=0.05$.
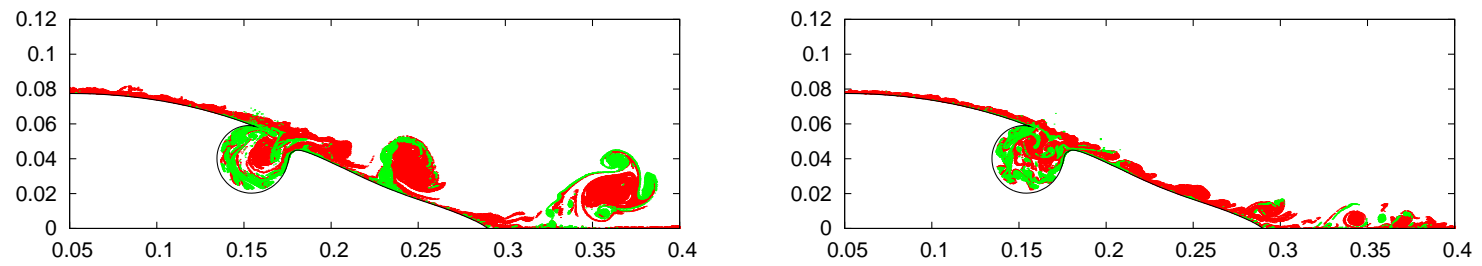

Figure 13: Vorticity distribution for constant suction $\left(C_{q}=0.03\right)$ (left) and oscillatory suction with $A_{0}=0.03, A_{1}=0.015$ and $T=0.1$ (right). Green denotes regions with positive vorticity and red negative vorticity.

The flow has been successfully stabilized with open-loop actuation and $T=0.1$. However, this actuation is at $300 \mathrm{~Hz}$ and practice it would be difficult to generate actuation at this frequency (this will be discussed further below). Hence a secondary aim is to reduce the frequency as low as possible while maintaining control of the flow.

Figure 14 shows a typical vorticity distributions with suction varying between $C_{q}=0$ and $C_{q}=0.03$ with $T=0.4,0.8,1.2$ and 1.5. The flow is still under control with a period of $T=1.2$, but is showing large scale vortex shedding with $T=1.5$. The flow would be expected to show large scale instabilities with suction dropping to zero if the period of the oscillation is large enough. However, the frequency at which this occurs is well below the characteristic shedding frequency $\left(4 U_{0} / L\right.$, i.e. $\left.T=0.25\right)$ for uncontrolled flow (Figure 12).

Figures 13 and 14 show typical vorticity distributions for flow with actuation at frequencies above and below that at which the major part of the unsteady disturbances occur, as shown by the power spectra of Figure 12. A number of calculations were performed with $T=0.25$, corresponding to the peak in Figure 12. Not surprisingly, the flow could not be reliably controlled with oscillatory actuation of this frequency using $A_{0}=0.03$ and $A_{1}=0.015$, as in Figures 13 and 14 .

\section{Experiments: Apparatus}

A number of experiments have been performed on the testbed model mounted in the CT- 1 wind tunnel at CIRA. The main aim for these experiments was to generate data in order to develop a larger model $(0.5 \mathrm{~m}$ chord) to be used in a different series of experiments in a much bigger wind tunnel (3x5m working section), with constant not variable suction as a control mechanism [17]. However, a short series of experiments was also planned using active control with suction with characteristics similar to those used in the simulations reported in previous sections of this paper in terms of volumetric flow rate and frequency 

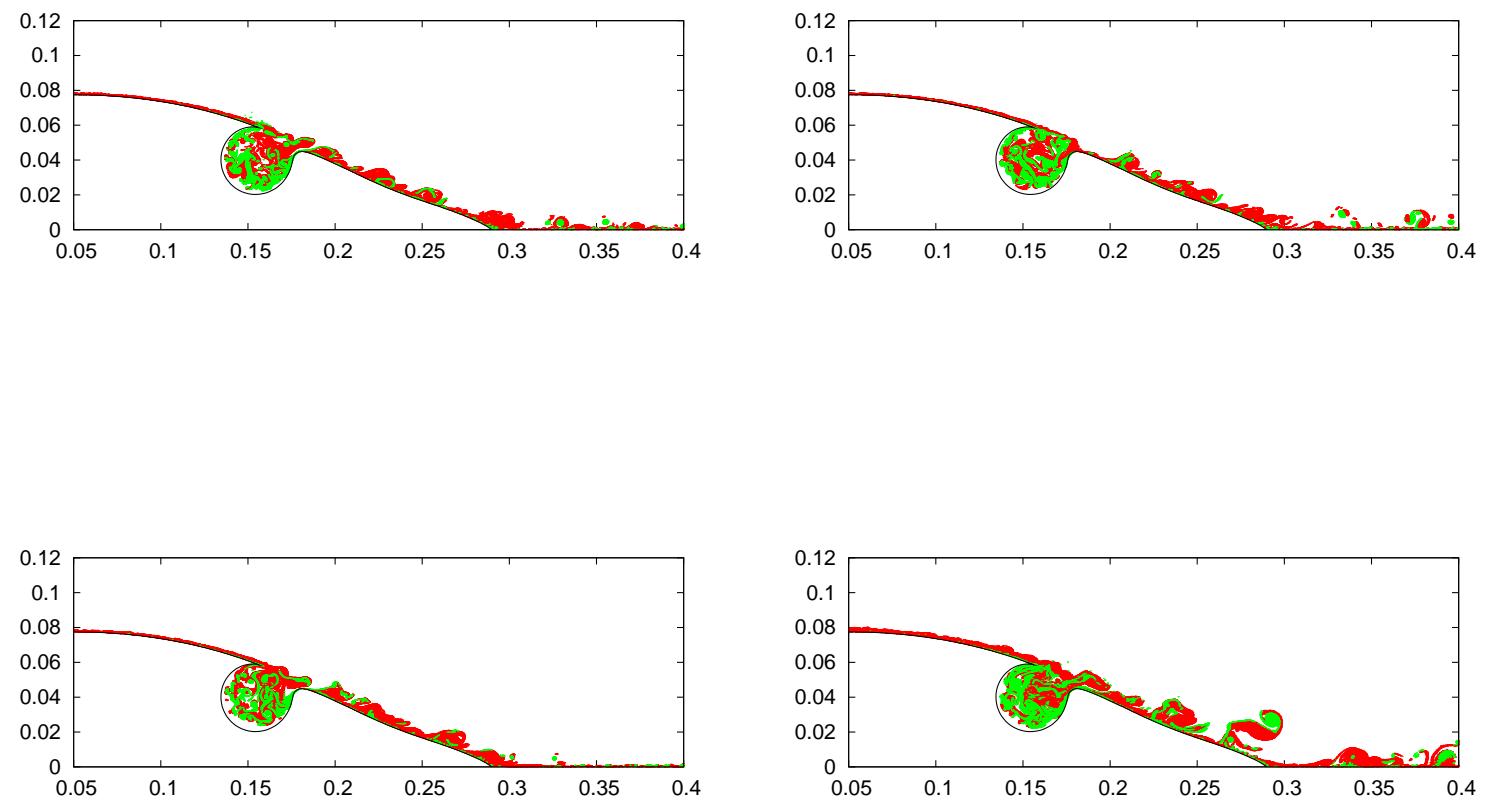

Figure 14: Vorticity distribution for oscillatory suction with $A_{0}=0.03, A_{1}=0.015$ and $T=0.4$ (top left), $T=0.8$ (top right), $T=1.2$ (bottom left) and $T=1.5$ (bottom right).

response. This did not prove possible due to the lack of variation in the flow rate at higher frequencies and the limited time available in the wind tunnel for the experiments. The experiments actually undertaken did, however, provide useful data to compare with that from the simulations, including the behavior with steady low frequency of oscillation suction. This section gives the relevant details of the tunnel used, with the experimental results obtained given and discussed in the following one.

The wind tunnel is of the open circuit form, with test section size of $0.305 \times 0.305 \times 0.6 \mathrm{~m}^{3}$, a maximum speed of $55 \mathrm{~m} / \mathrm{s}$, a nozzle contraction rate of $16: 1$, and a maximum value of the turbulence level at $50 \mathrm{~m} / \mathrm{s}$ flow speed of $0.1 \%$. The bottom of the wind tunnel test section was modified in order to accommodate the model and allow access for the suction system. The model mounted on the lower wall is shown in Figure 15 .

The flow over an airfoil mounted on the bottom of the tunnel as in Figure 15 will naturally have a large separation/recirculation region immediately upstream of the model. This region of the flow will be highly unstable, generating significant large scale unsteadiness in the incoming flow. In order to reduce the separation and eliminate this unwanted disturbance, suction panels with a porosity of $9.8 \%$ were placed on the lower wall of the tunnel immediately upstream of the model, under its leading edge (Figure 15). Constant suction through these panels was successful in stabilizing the flow in the region of the leading edge and upstream of the airfoil.

The model represents a two dimensional airfoil with a chord length of $0.35 \mathrm{~m}$ and a span of $0.305 \mathrm{~m}$. The model angle of attack (AoA) can range between $5.66^{\circ}$ to $12.66 \circ$ with a step of $1^{\circ}$. The cavity shape has been designed starting from the data of the pressure surface distribution measured on a clean airfoil 


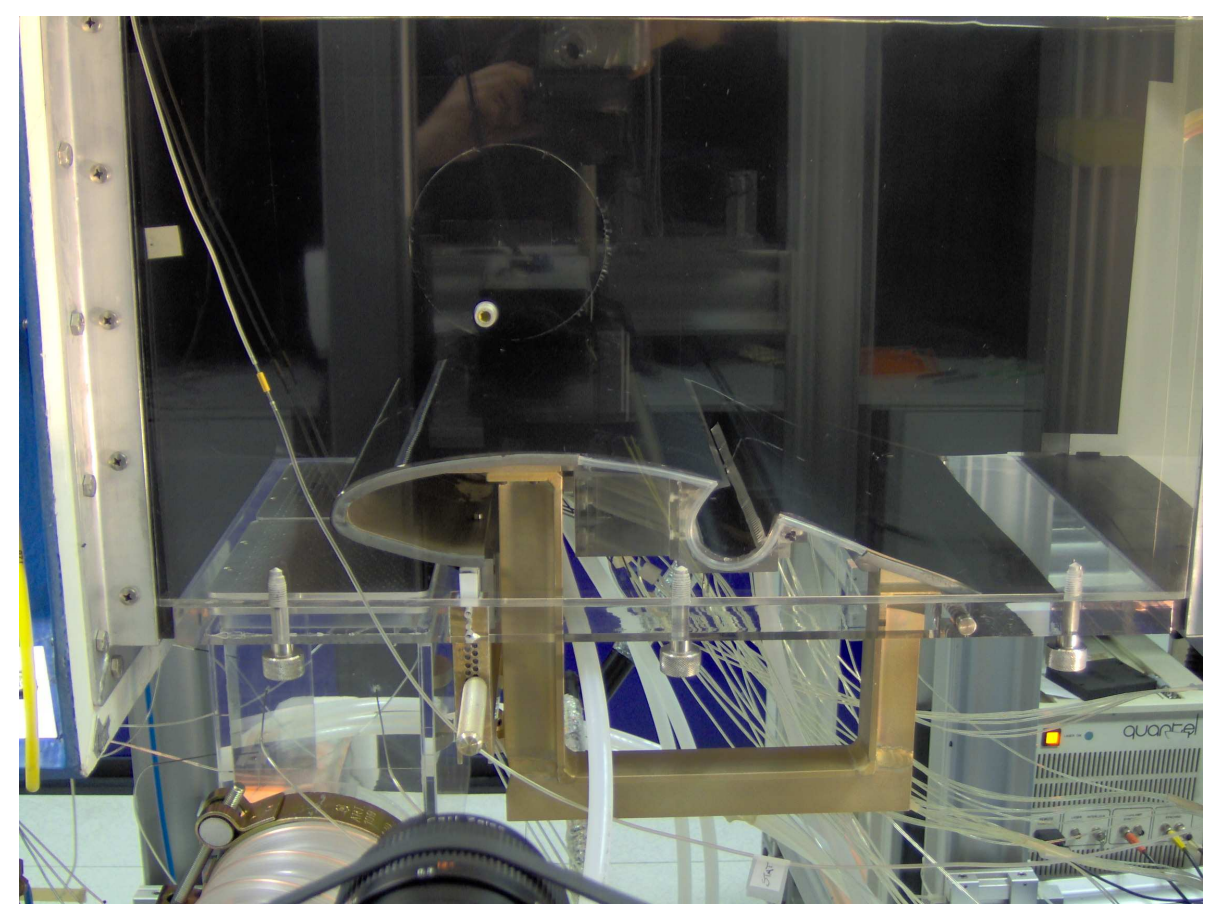

Figure 15: Test bed model mounted on the lower wall of the wind tunnel.

(i.e. one without a cavity), using a numerical potential code as described in [18].

The suction panels on the leading edge of the cavity consist of 906 holes with a diameter of $1 \mathrm{~mm}$, clustered into three groups (CAV1-CAV3), as shown in Figure 16. The holes are connected to three different collectors, which are then connected to a vacuum pump. Each circuit has an electronically controllable valve (FESTO MPYE5-3/8-010-B), so that the suction flow rate to each panel/cluster of holes can be adjusted independently. The volumetric flow rate to each panel is obtained from efector300 flow meters placed between the valves and the pump. There is also a blowing panel, consisting of 126 holes, near the downstream lip to the cavity (CAV4, Figure 16). However, this was not used for the experiments and simulations described here, which considered suction only, in an attempt to make the control system as simple as possible. The three pipes for the suction system can be seen in Figure 15 (the large diameter pipes leading vertically downwards from the model).

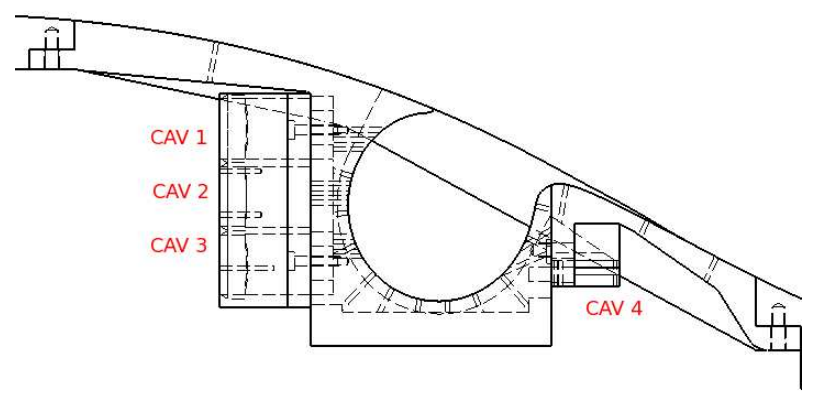

Figure 16: Trapped vortex cavity, showing the three suction panels (CAV1-CAV3) and the blowing panel (CAV4). 
The model is equipped with 37 pressure taps, 33 along the chord of the model as shown in Figure 17, and 4 spanwise in the cavity. The mean values of the surface pressure on the model and the static pressure upstream of the model have been acquired through a Hyscan 2000 system. The pressure taps are connected to a Scanivalve ZOC 22B electronic pressure scanner, characterized by: full scale ranges of \pm 1 psid, an accuracy value of $\pm 0.15 \%$ F.S., scan rate of $20 \mathrm{kHz}$ and a temperature sensitivity of $0.05 \%$ F.S. $/{ }^{\circ}$ C. Some of the tubes running from the surface of the model to the scanner can be seen in Figure 15 (the small diameter tubes in the bottom left of the photograph).

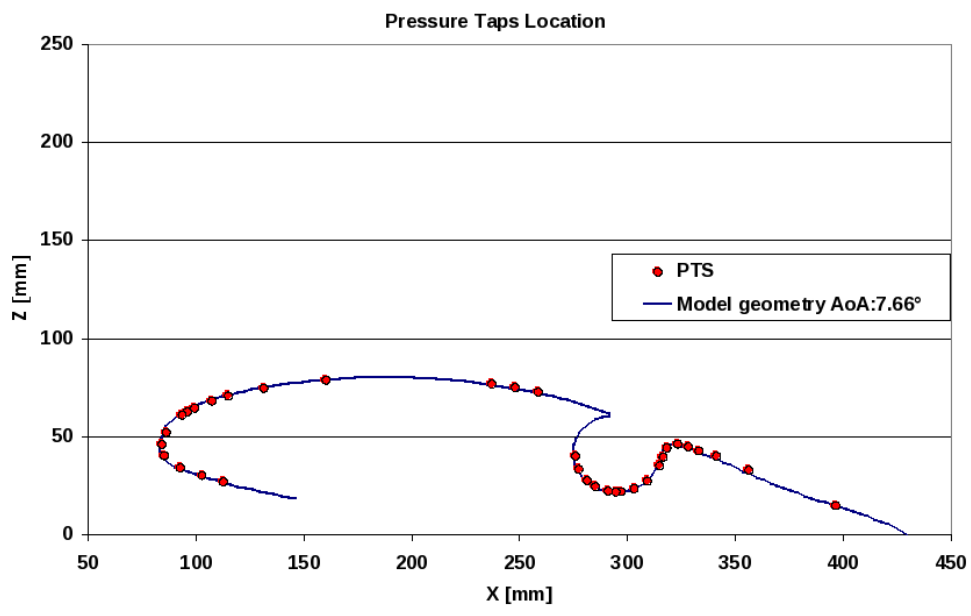

Figure 17: Model geometry showing the position of the pressure taps.

The model is constructed from transparent material to allow the use of optical measurement techniques, in particular Particle Image Velocimetry (PIV). The recording region is illuminated by two Nd-Yag resonator heads providing a laser beam energy of about $300 \mathrm{~mJ}$ each at a wave length of $532 \mathrm{~nm}$. In order to measure simultaneously the upper and lower region of the model, and in particular, to illuminate the flow field inside the cavity, a double light sheet configuration has been adopted. The laser beam, after the recombination optics, is split into two beams. The beams are directed inside two different mechanical arms, each composed of seven mirrors and able to provide six freedom degrees. The arms can deliver the beams up to a distance of 2 meters from the laser. At the exit of the arms a set of lens (spherical and cylindrical) opens the beam in a light sheet which can be focused at the required distance.

The recording system consists of two high resolution CCD cameras (2048x2048 pixels). One camera, mounted with a $100 \mathrm{~mm}$ lens, provides a high resolution image $(0.5 \mathrm{~mm} /$ vector $)$ over a measurement area of $65 \times 65 \mathrm{~mm}^{2}$. This camera was used for measuring the flow field inside the cavity and close to the model surface. It was mounted on a remotely controllable, two-dimensional linear transversing system. The second camera was mounted on an optical bench further from the model. It has a $60 \mathrm{~mm}$ lens, and acquired data in a larger region of approximately 270x270 $\mathrm{mm}^{2}$, characterized by a spatial resolution of $2.1 \mathrm{~mm} /$ vector. It was used to measure the external flow above the full model.

\section{Experiments: Results}

Figure 18 shows the flow rates through the suction panels as a function of the control voltage to the valves, varying between 0 volts (fully open) and 5 volts (closed). Clearly the suction flow rate is a highly non-linear function of the control voltage, with most of the variation in the flow rate occurring in the range of 3-5 volts. The total maximum flow rate is approximately $25 \mathrm{~m}^{3} /$ hour, which corresponds to a 
mean suction velocity on the panels of $1.4 \mathrm{~m} / \mathrm{s}$.

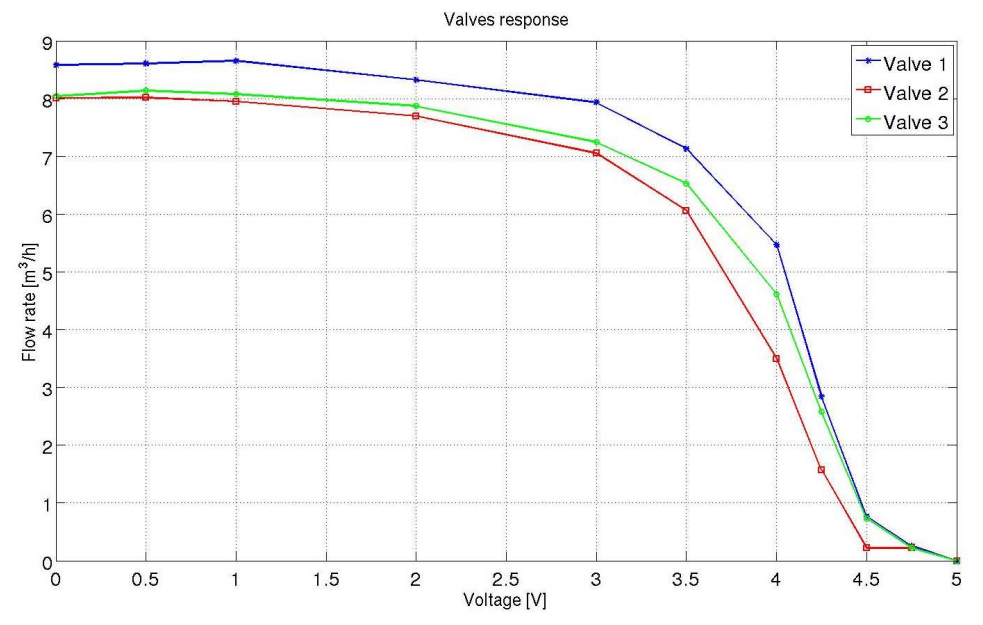

Figure 18: Steady state volumetric flow rate to the suction panels as a function of the control voltage to the valves.

The simulations reported above have been performed with the model at $7^{\circ}$ AoA, which was the original design point for the experiments. However, due to manufacturing difficulties, the model could not be placed at $7^{\circ}$. Instead, the main body of the experiments with control were with the model at $7.66^{\circ}$ AoA.

The pressure tappings on the model produced values of the mean pointwise surface pressure, but no information about the unsteady behavior. Hence these cannot be used to implement an active control scheme such as the PI controller described above. However, the mean surface pressure values can be used to determine if the mean flow is attached or separated. Figure 19 shows the pressure coefficient (pressure normalized by $\left.\frac{1}{2} \rho U_{0}^{2}\right)$ along the surface of the model for zero suction $\left(C_{q}=0\right)$, and total suction with $Q_{T} \approx 21.1 \mathrm{~m}^{3} / \mathrm{h}\left(C_{q}=0.08\right)$, corresponding to control voltages of 0 and 4.2 volts, respectively. The model is at $7.66^{\circ}$ AoA with a flow with $U_{0}=15 \mathrm{~m} / \mathrm{s}$. Upstream of the cavity $(x / c<0.5$ where $c$ is the chord of the model) there is little variation in the pressure distribution, but a significant change in the cavity $(x / c \approx 0.6)$, and on the model downstream of the cavity $(x / c>0.7)$.

A flat pressure distribution as shown in Figure 19 downstream of the cavity when there is no suction is characteristic of separated flow. Figure 20 shows PIV (Particle Image Velicometry) measurements and streamlines of the mean flow for the no suction case. The streamlines clearly show the region of separation downstream of the cavity. Note that the regularity of the streamlines of Figure 20 arises from the averaging over time performed to generate the mean flow; instantaneous snapshots of the flow would show much more irregular behavior, including shedding of vortices from the region of the cavity, this can be seen in Figure 26 below.

Figure 21 shows PIV measurements and streamlines for the case with suction in Figure 19. In contrast to the zero suction case (Figure 20), the streamlines show that the mean flow is attached downstream of the cavity.

The suction successfully suppresses the separation of the flow downstream of the cavity when $U_{0}=15$ $\mathrm{m} / \mathrm{s}$ (Figures 19 and 21). In contrast, when the free stream velocity is $30 \mathrm{~m} / \mathrm{s}$, there is little difference between the pressure coefficient with zero and maximum suction (Figure 22). In both cases the mean flow is separated, although with a larger separation region in the zero suction case, as shown by the streamlines for the mean flow, see Figure 23.

In the experiments with the model at $7.66^{\circ}$ AoA, the suction provided sufficient leverage on the flow to maintain attached flow with a free stream velocity of $15 \mathrm{~m} / \mathrm{s}$ but not with $30 \mathrm{~m} / \mathrm{s}$. This observation 


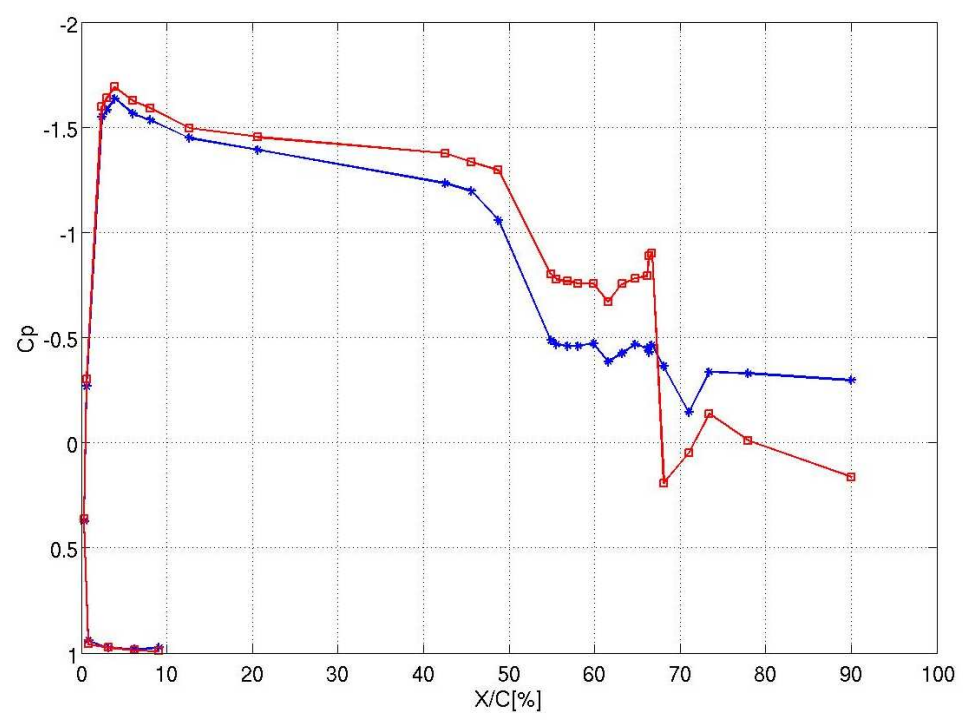

Figure 19: Pressure coefficient for the mean surface pressure on the model at $7.66^{\circ}$ AoA and $U_{0}=15 \mathrm{~m} / \mathrm{s}$. At the right, the top (blue/*) line has zero suction and the bottom (red/squares) line has $Q_{T} \approx 21.1 \mathrm{~m}^{3} / \mathrm{h}$.

provides a primary point of comparison between the results of the experiments and the DVM simulations. In the simulations with the model at $7^{\circ}$ as reported above, suction with $C_{q}=0.05$ was sufficient to maintain attached flow with $U_{0}=30 \mathrm{~m} / \mathrm{s}$. A further set of calculations were performed with the model at $7.66^{\circ}$. In these, a higher suction flow rate with $C_{q} \approx 0.08$ was required to maintain attached flow for both $U_{0}=15 \mathrm{~m} / \mathrm{s}$ and $U_{0}=30 \mathrm{~m} / \mathrm{s}$. A suction coefficient of 0.08 corresponds to a volumetric flow of approximately $21 \mathrm{~m}^{3} / \mathrm{h}$ with $U_{0}=15 \mathrm{~m} / \mathrm{s}$ and $42 \mathrm{~m}^{3} / \mathrm{h}$ with $U_{0}=30 \mathrm{~m} / \mathrm{s}$. Thus, there is agreement with the experiments in that the simulations predict that it should be possible to maintain attached flow with the model at $7.66^{\circ}$ and with $U_{0}=15 \mathrm{~m} / \mathrm{s}$ but not with $U_{0}=30 \mathrm{~m} / \mathrm{s}$.

In general, at relatively low AoA, as the AoA of an airfoil is increased the point of separation will move forward. Since the position of the cavity is fixed, this increase in AoA would be expected to make it more more difficult to control the flow, as was found in both the simulations and the experiments. Some simulations were also performed with oscillatory flow and the model at $7.66^{\circ}$. Again, the flow was more difficult to control, with the unsteadiness of the actuation having much less effect than when the model was at $7^{\circ}$. However, another, more fundamental problem, was encountered when attempting to apply oscillatory flow in the experiments. The valves were quoted as operating up to $60 \mathrm{~Hz}$ in the manufacturers specifications. However, the system is highly non-linear, and the inertia in the system was such that the variation in the volumetric flow rate was much lower than would be expected from the volumetric flow rate for steady suction. In fact, except at very low frequencies (below $5 \mathrm{~Hz}$ ), there was only a small variation in flow rate through the pipes, even with a relatively large oscillation in the input voltage. For example, Figure 24 shows the volumetric flow rate for each of the suction panels for a triangular signal, varying between 3.2 and 4.6 volts for valve 1 , and 2.3 and 4.5 volts for valves 2 and 3. Although the mean flow rate is consistent with that expected (Figure 18), clearly the variation is much smaller than expected and will have little effect on the flow. This was confirmed by examination of the PIV data for a large number of runs with different time varying signals with a frequency of $5 \mathrm{~Hz}$ or above.

However, at lower frequencies there is a significant variation in the flow rates. Figure 25 shows the flow rates for the suction panels for a square wave signal varying between 2.5 and 4.9 volts for valve 1 , 

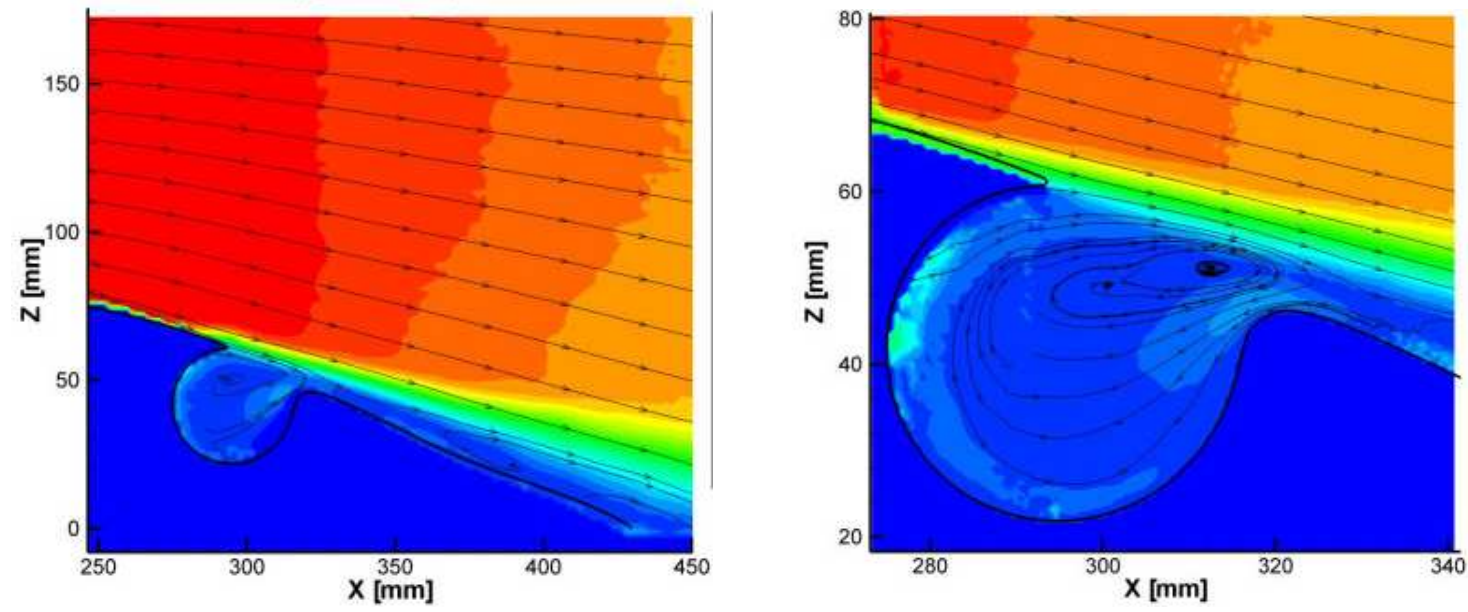

Figure 20: PIV measurements and streamlines for flow past the model at $7.66^{\circ}$ AoA with $U_{0}=15 \mathrm{~m} / \mathrm{s}$ and no suction. The color gives the magnitude of the velocity and the solid lines the streamlines derived from the experimental velocity field. The lengths are in $\mathrm{mm}$.

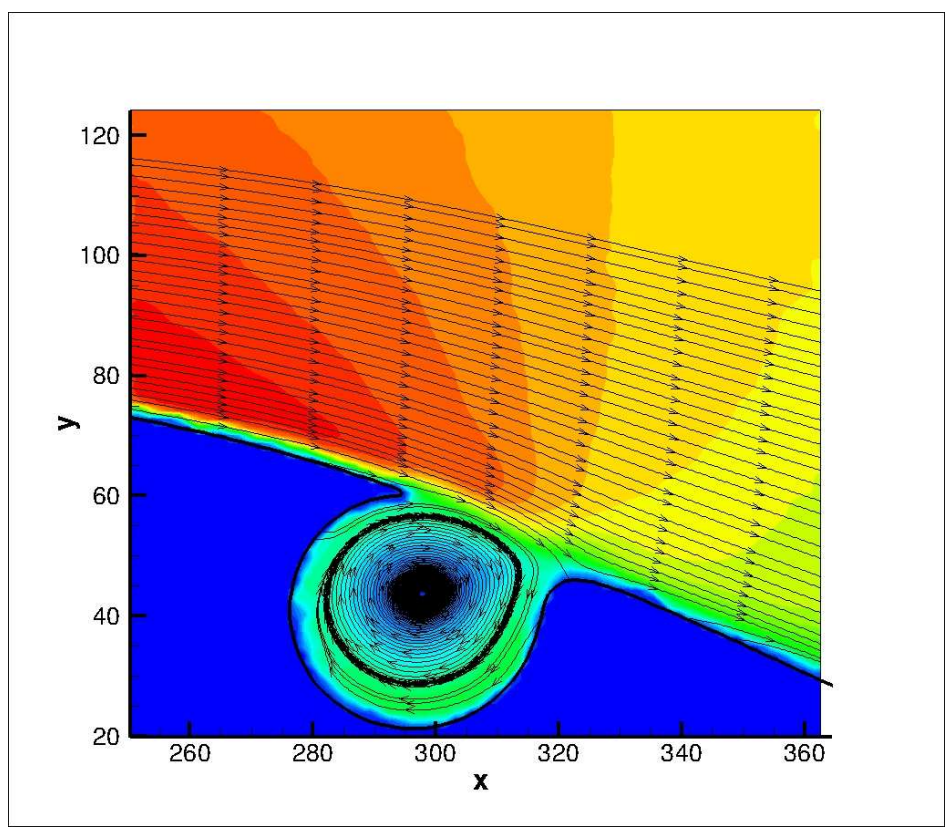

Figure 21: PIV measurements and streamlines for flow past the model at $7.66^{\circ}$ AoA with $U_{0}=15 \mathrm{~m} / \mathrm{s}$ and $Q_{T} \approx 21.1 \mathrm{~m}^{3} / \mathrm{h}$. 


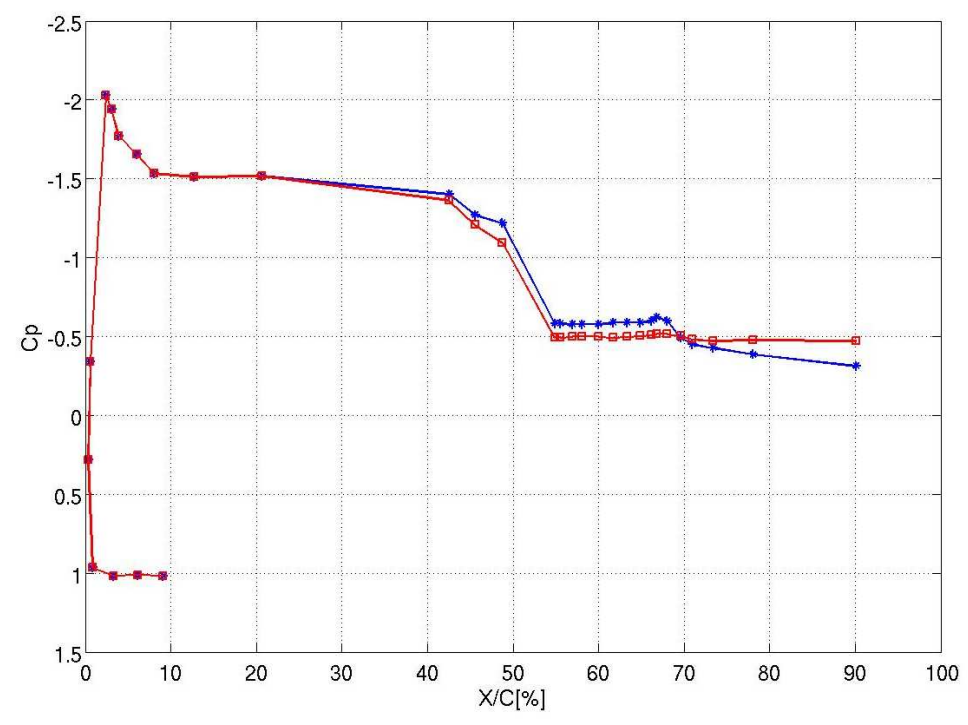

Figure 22: Pressure coefficient for the mean surface pressure on the model at $7.66^{\circ}$ AoA and $U_{0}=30 \mathrm{~m} / \mathrm{s}$ for zero suction $(X)$ and strong suction with $Q_{T} \approx 24.5 \mathrm{~m}^{3} / \mathrm{s}(+)$.

between 2.34 and 4.74 volts for valve 2 , and 2.38 and 3.78 volts for valve 3 . Figure 26 shows typical PIV snapshots taken at the four points in the cycle marked on Figure 25. There is a marked difference in the flow when the suction is increasing as compared to when it is decreasing, with essentially attached flow during the former and separated flow during the latter. As the suction flow rate increases, more fluid is drawn into the cavity from the flow past the mouth of the cavity. Hence the flow from upstream of the cavity which will impinge on the wall at and downstream of the lower lip of the cavity will have higher momentum and be more resistant to separation. As the suction flow rate decreases, the reverse happens, and the flow will become more prone to separation, as illustrated in Figure 26. Note that a frequency of $1 \mathrm{~Hz}$ is well below the frequency found necessary to suppress the vortex shedding in the simulations (a non-dimensional period of 1.2 corresponds to a frequency of $12.5 \mathrm{~Hz}$ with $U_{0}=15 \mathrm{~m} / \mathrm{s}$ ).
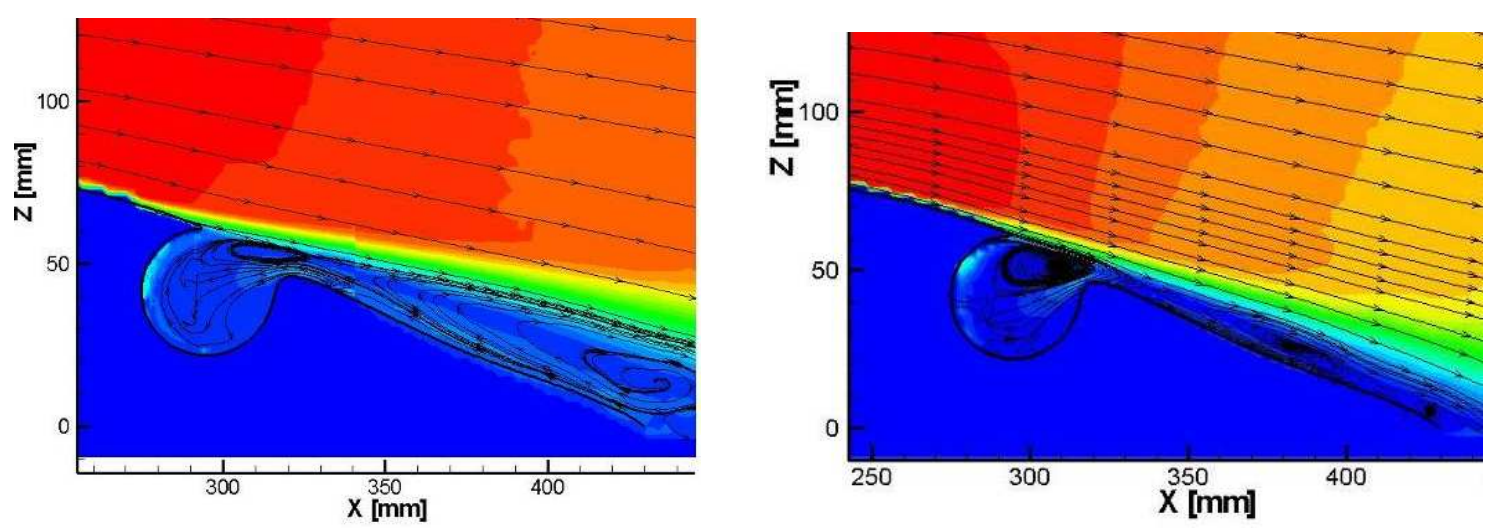

Figure 23: PIV measurements and streamlines for flow past the model at $7.66^{\circ}$ AoA with $U_{0}=30 \mathrm{~m} / \mathrm{s}$ and no suction (left) and $Q_{T} \approx 24.5 \mathrm{~m}^{3} / \mathrm{h}$ (right). 


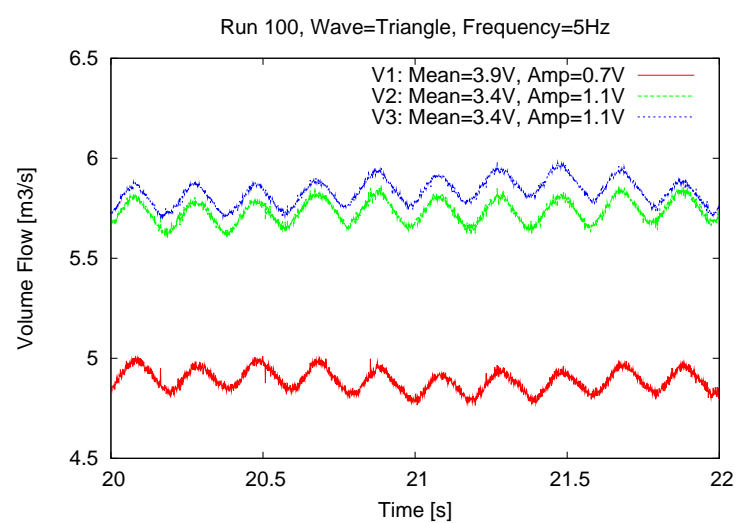

Figure 24: Volumetric flow rates for the valves with a triangular signal at $5 \mathrm{~Hz}$. The signal for valve 1 varies between 3.2 and $4.6 \mathrm{~V}$, and for valves 2 and 3, between 2.3 and $4.5 \mathrm{~V}$.

\section{Conclusions}

In this work we have investigated methods of trapping a vortex in a cavity so that the flow remains attached with no large scale vortex shedding. A DVM model of the flow has been used to develop simple but effective control strategies. In particular, a feasible strategy is to use constant suction or a PI controller to stabilize the flow, followed by oscillatory open loop suction to maintain stability with a significantly reduced effort, and at a much lower frequency than the characteristic vortex shedding frequency from the uncontrolled flow.

An attempt has been made to realize this strategy in wind tunnel experiments. This was only partially successful due to a number of reasons. In particular, we were not able to source a set of valves with the required characteristics, i.e. ones capable of generating a sufficiently large variation in the suction flow rates at the frequency required, while it was not possible to produce a more complex/sophisticated system within the resources available to the project. However, there was sufficient agreement between the results from the simulations and the experiments (critical suction flow rate required to stabilize the flow, and lack of control at low frequencies of actuation) to suggest the control strategy developed is viable.

\section{Acknowledgments}

This work was supported through an EU FP6 grant, VortexCell2050: Fundamentals of actively controlled flows with trapped vortices (AST-CT-2005-012139).

\section{References}

[1] Ringleb, F.O. (1961) Separation control by trapped vortices. In: Boundary Layer and Flow Control, ed. G.V. Lachman, Pergamon Press.

[2] Kasper, W.A. (1975) Some ideas of vortex lift. Society of Automative Engineers, paper 750547, $12 \mathrm{pp}$.

[3] Kruppa, E.W. (1977) A wind tunnel investigation of the Kasper vortex concept. AIAA paper 115704, 10pp. 


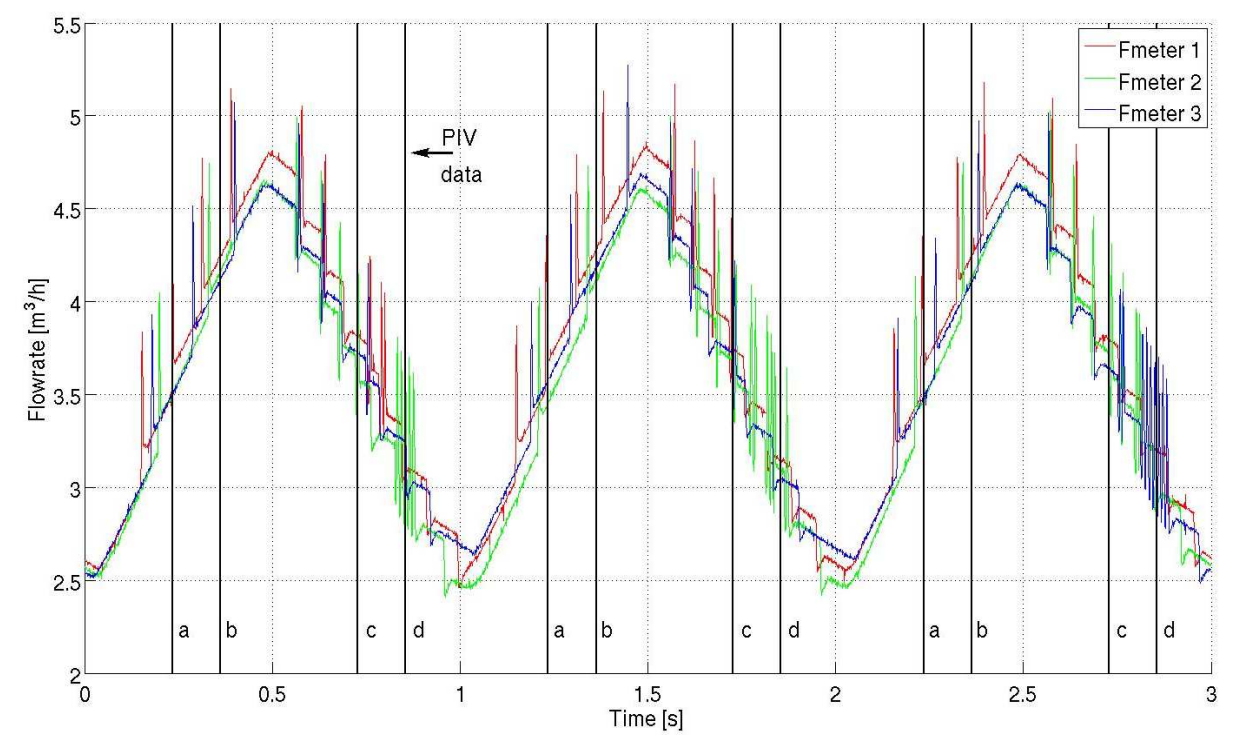

Figure 25: Volumetric flow rates for a square signal at $1 \mathrm{~Hz}$. The signal for valve 1 varies between 2.5 and $4.9 \mathrm{~V}$, between 2.34 and $4.74 \mathrm{~V}$ for valve 2 , and 2.38 and $4.78 \mathrm{~V}$ for valve 3 . The vertical lines show the points that PIV snapshots were taken.

[4] http://www.ekip-aviation-concern.com/

[5] Hackenberg, P., Rioual, J.L., Tutty, O.R. \& Nelson, P. (1995) The automatic control of boundary layer transition - experiments and computation. Applied Scientific Research, 54, 293-311.

[6] Veres, G.V., Tutty, O.R., Rogers, E. \& Nelson, P.A. (2004) Global optimisation-based control algorithms applied to boundary layer transition problems. Control Engineering Practice, 12, 475490.

[7] Cottet, G.-H. \& Koumoutsakos, P.D. (2000) Vortex Methods: Theory and Practice. Cambridge University Press.

[8] Clarke, N.R. \& Tutty, O.R. (1994) Construction and Validation of a discrete vortex method for the two-dimensional incompressible Navier-Stokes equations. Computers \& Fluids, 23, 751-783.

[9] Shankar, S. \& van Dommelen, L. (1996) A new diffusion procedure for vortex methods. J. Comput. Phys., 127, 88-109.

[10] Takeda, K., Tutty, O.R. \& Fitt, A.D. A comparison of four viscous models for the discrete vortex method. AIAA 13th Computational Fluid Dynamics Meeting, Colorado, July 1997. AIAA paper 97-1977, 11pp.

[11] Batchelor, G.K. An Introduction to Fluid Dynamics, Cambridge University Press, 1967.

[12] Anderson, J.D. Fundamentals of Aerodynamics, Singapore, McGraw-Hill, 1985.

[13] Métais, O. and Lesieur, M. (1992) Spectral large-eddy simulations of isotropic and stably-stratified turbulence. J. Fluid Mech., 239, 157-194.

[14] Lesieur, M. and Métais, O. (1996) New trends in large-eddy simulations of turbulence. Ann. Rev. Fluid Mech., 28, 45-82. 

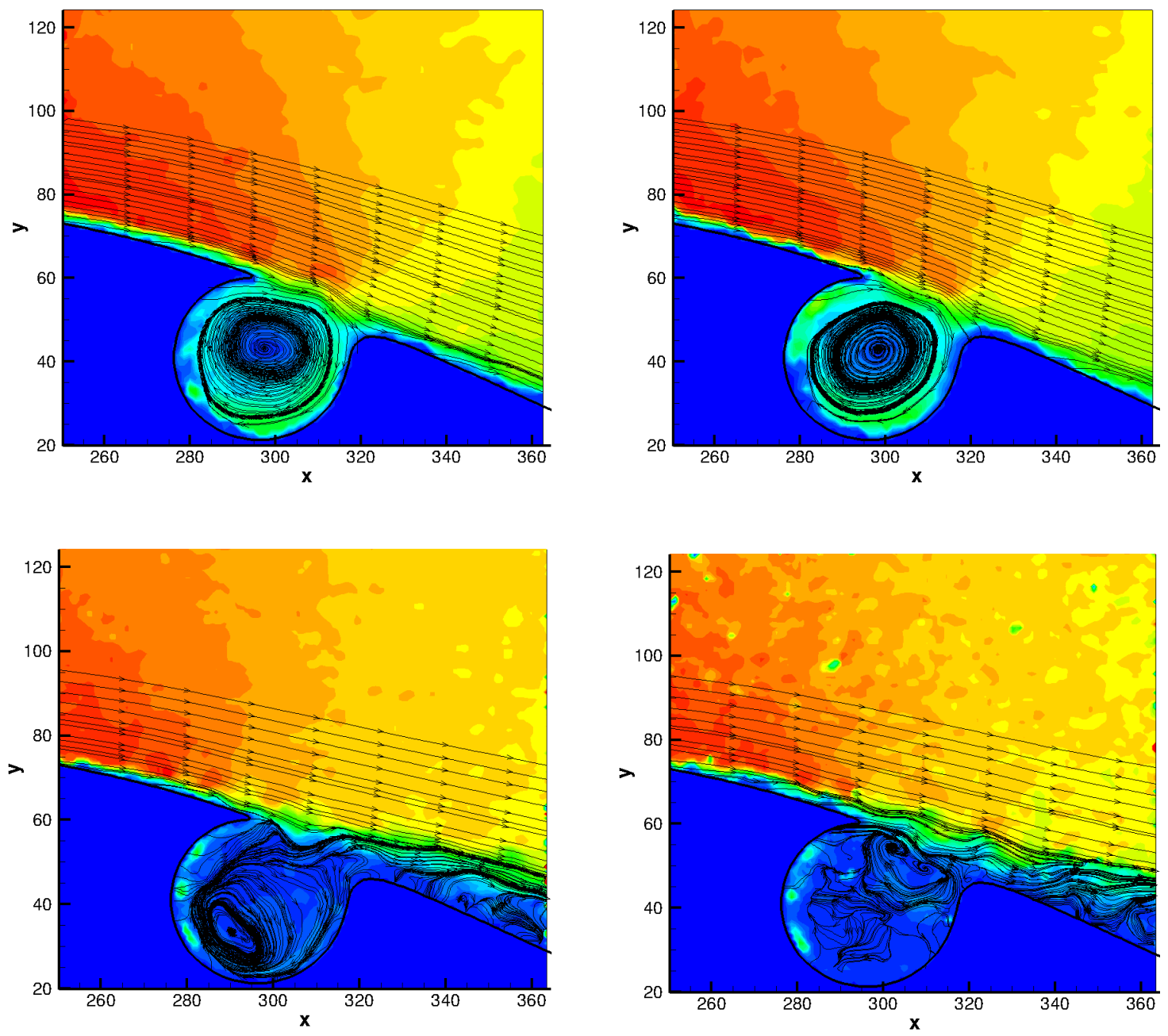

Figure 26: Instantaneous PIV images for flow past the model at $7.66^{\circ}$ with $U_{0}=15 \mathrm{~m} / \mathrm{s}$. The images are taken at different times in the cycle, as marked on Figure 25: (a) top left, (b) top right, (c) bottom left, (d) bottom right. 
[15] Iannelli, P. (2006) RANS-based aerodynamics analysis of the flow separation test-bed installed in the CIRA CT1 wind tunnel. Tech. Report AST4-CT-2005-012139-D3.1.3. Centro Italiano Richerche Aerospaziali, Italy.

[16] Astom, K. J. \& Hagglund, T. (1995) PID controllers: Theory Design and Tuning Instrument Society of America.

[17] Lasagna, D., Donelli, R., De Gregorio, F. \& Iuso, G. (2011) Effects of a trapped vortex cell on a thick wing airfoil. Exp. Fluids, 51, 13691384.

[18] Hetsch, T., Savelsberg, R., Chernyshenko, S.I. \& Castro, I.P. (2009) Fast numerical evaluation of flow fields with vortex cells. European Journal of Mechanics B - Fluids, 28, 660-669. 